\title{
STRONG CONVERGENCE OF A FULLY DISCRETE FINITE ELEMENT APPROXIMATION OF THE STOCHASTIC CAHN-HILLIARD EQUATION
}

\author{
DAISUKE FURIHATA, MIHÁLY KOVÁCS, STIG LARSSON, AND FREDRIK LINDGREN
}

\begin{abstract}
We consider the stochastic Cahn-Hilliard equation driven by additive Gaussian noise in a convex domain with polygonal boundary in dimension $d \leq 3$. We discretize the equation using a standard finite element method in space and a fully implicit backward Euler method in time. By proving optimal error estimates on subsets of the probability space with arbitrarily large probability and uniform-in-time moment bounds we show that the numerical solution converges strongly to the solution as the discretization parameters tend to zero.
\end{abstract}

\section{INTRODUCTION}

Let $\mathcal{D} \subset \mathbb{R}^{d}, d \leq 3$, be a convex spatial domain with polygonal boundary $\partial \mathcal{D}$ and consider the stochastic Cahn-Hilliard equation, also known as the Cahn-HilliardCook equation [4, 8, 9, written in the abstract Itô form

$$
\mathrm{d} X+A(A X+f(X)) \mathrm{d} t=\mathrm{d} W, t \in(0, T] ; \quad X(0)=X_{0},
$$

where $-A$ is the Laplacian with homogeneous Neumann boundary conditions and where $\{W(t)\}_{t \geq 0}$ is an $H:=L_{2}(\mathcal{D})$-valued $Q$-Wiener process on a filtered probability space $\left(\Omega, \mathcal{F}, \mathbf{P},\left\{\mathcal{F}_{t}\right\}_{t \geq 0}\right)$ with respect to the normal filtration $\left\{\mathcal{F}_{t}\right\}_{t \geq 0}$. In order to avoid additional technical difficulties we assume that the initial-value $X_{0}$ is deterministic. For the weak (and mild) solution $X$ (see, Theorem 3.1) to preserve mass, we assume that the average $|\mathcal{D}|^{-1} \int_{\mathcal{D}} W(t) \mathrm{d} x=0$ for all $t \geq 0$.

The nonlinear function $f$ is assumed to be of the form $f=F^{\prime}$, where $F$ has the following structural property:

(1.2) $F$ is a polynomial of degree 4 with leading term $c_{0} s^{4}$ where $c_{0}>0$.

A typical example is $F(s)=\frac{1}{4}\left(s^{2}-1\right)^{2}$, which is a double well potential. Note that $f$ is only locally Lipschitz and does not satisfy a linear growth condition. We also note that the restriction on the polynomial degree of $F$ comes from the fact that we allow $d=3$. For $d=1,2$ the exponent 4 in (1.2) may be replaced by any even integer larger than or equal 4 and the arguments of the paper are still valid

2000 Mathematics Subject Classification. 60H15, 60H35, 65C30.

Key words and phrases. stochastic partial differential equation; Cahn-Hilliard-Cook equation; additive noise; Wiener process; Finite element method, Euler method; time discretization; strong convergence.

M. Kovács was supported by Marsden Fund of the Royal Society of New Zealand project number UOO1418.

F. Lindgren was supported by JSPS KAKENHI grant number $15 \mathrm{~K} 45678$ and by The Swedish Foundation for International Cooperation in Research and Higher Education, STINT through the Capstone Award. 
with trivial changes. It is easy to see that (1.2) implies the following dissipativity property, with $\langle\cdot, \cdot\rangle,\|\cdot\|$ denoting the scalar product and norm in $H=L_{2}(\mathcal{D})$,

$$
\langle f(v), v\rangle \geq-C_{0}, \quad v \in L_{4}(\mathcal{D})
$$

for some $C_{0}$. It also follows that $F^{\prime \prime}(s) \geq-c_{1}^{2}$ for some $c_{1}$, which yields

$$
F(x)-F(y) \leq f(x)(x-y)+\frac{1}{2} c_{1}^{2}(x-y)^{2}, \quad x, y \in \mathbf{R} .
$$

Finally, as $f$ is a polynomial of degree 3 we have, for some $C>0$, that

$$
|f(x)-f(y)| \leq C\left(1+x^{2}+y^{2}\right)|x-y|, \quad x, y \in \mathbf{R} .
$$

It is not hard to see that, due to the homogeneous Neumann boundary conditions and since the average of $W$ equals 0 , it follows that $X$ preserves the average (the total mass), that is, $|\mathcal{D}|^{-1} \int_{\mathcal{D}} X(t) \mathrm{d} x=|\mathcal{D}|^{-1} \int_{\mathcal{D}} X_{0} \mathrm{~d} x$, cf. Remark 5.1 Note that for $s_{0} \in \mathbb{R}$ the function $\tilde{F}(s)=F\left(s+s_{0}\right)$ also has the structural property (1.2). Therefore, one can employ a change of variables $X \rightarrow X-|\mathcal{D}|^{-1} \int_{\mathcal{D}} X_{0} \mathrm{~d} x$, and hence we will assume that the average $|\mathcal{D}|^{-1} \int_{\mathcal{D}} X_{0} \mathrm{~d} x=0$.

We fix a finite time horizon $T>0$ and for $N \in \mathbb{N}$ consider the fully implicit finite element method

$$
\begin{aligned}
X_{h}^{j}-X_{h}^{j-1}+k A_{h}^{2} X_{h}^{j}+k A_{h} P_{h} f\left(X_{h}^{j}\right) & =P_{h} \Delta W^{j}, \quad j=1,2, \ldots, N, \\
X_{h}^{0} & =P_{h} X_{0} .
\end{aligned}
$$

Here $k=T / N$ is the time-step, $t_{j}=j k, \Delta W^{j}=W\left(t_{j}\right)-W\left(t_{j-1}\right),-A_{h}$ is the discrete Laplacian, and $P_{h}$ is the orthogonal projector onto the finite element space $S_{h}$ with mesh size $h>0$; for more details on the finite element method, see Section 2.2. It is easy to see that also $X_{h}^{j}$ preserves the mass, cf. Remark 5.1 An implementation based on the open source finite element software FEniCS can be found in http://www. math. chalmers. se/\%7estig/code/chc. py.

The main result of the paper, Theorem [5.6] asserts that if the operator composition $A^{\frac{1}{2}} Q^{\frac{1}{2}}$ is Hilbert-Schmidt and the initial data is regular enough; that is, for some $L>0$,

$$
\left|X_{h}^{0}\right|_{1}+\mathcal{F}\left(X_{h}^{0}\right)+\left|A_{h} X_{h}^{0}+P_{h} f\left(X_{h}^{0}\right)\right|_{1}+\left|X_{0}\right|_{1} \leq L \text { for all } h>0,
$$

where $\mathcal{F}(v)=\int_{\mathcal{D}} F(v) \mathrm{d} x$ and $|v|_{1}=\left\|A^{\frac{1}{2}} v\right\|=\|\nabla v\|$, then

$$
\lim _{h, k \rightarrow 0} \mathbf{E} \sup _{0 \leq n \leq N}\left\|X\left(t_{n}\right)-X_{h}^{n}\right\|^{2}=0 .
$$

The key result used in the proof is a maximal type moment bound on $\left|X_{h}^{j}\right|_{1}$, which is established in Theorem 4.3 after bootstrapping arguments. There are various difficulties in the proofs that are partly due to the finite element method. First, the finite element method is based on approximating the operator $A$ and not $A^{2}$. This is because the standard finite element functions belong only to the domain of $A^{\frac{1}{2}}$ but are not more regular. Loosely speaking this means that $A_{h}^{2} \neq\left(A^{2}\right)_{h}$, which makes already the deterministic finite element analysis more challenging. Second, the presence of the finite element projection $P_{h}$ in front of the semilinear term destroys some of the dissipativity properties of $f$. While $f$ enjoys the dissipativity property (1.3), and even

$$
\left\langle A^{\frac{1}{2}} f(v), A^{\frac{1}{2}} v\right\rangle=\langle\nabla f(v), \nabla v\rangle \geq-c|v|_{1}^{2},
$$

we only have

$$
\left\langle P_{h} f\left(v_{h}\right), v_{h}\right\rangle=\left\langle f\left(v_{h}\right), v_{h}\right\rangle \geq-C_{0}, \quad v_{h} \in S_{h},
$$


and unfortunately

$$
\left\langle A_{h}^{\frac{1}{2}} P_{h} f\left(v_{h}\right), A_{h}^{\frac{1}{2}} v_{h}\right\rangle=\left\langle A^{\frac{1}{2}} P_{h} f\left(v_{h}\right), A^{\frac{1}{2}} v_{h}\right\rangle \nsupseteq-c\left|v_{h}\right|_{1}^{2}, \quad v_{h} \in S_{h} .
$$

Because of the latter we can only establish a non-uniform moment bound on $\left\|X_{h}^{j}\right\|$ in Lemma 4.2 As

$$
\langle A f(x), A x\rangle \nsupseteq-c\|A x\|^{2} \text { and }\left\langle A_{h} P_{h} f\left(v_{h}\right), A_{h} v_{h}\right\rangle \nsupseteq-c\left\|A_{h} v_{h}\right\|^{2},
$$

the proof of the main moment bound in Theorem 4.3 is rather tedious and not entirely straightforward. In the proof we bound the discrete version of the Ljapunov functional for the original deterministic problem, see (4.15). Having maximaltype moment bounds at hand we use the mild formulation of both (1.1) and (1.6) to establish pathwise error bounds on subsets of the probability space with large probability in Theorem 5.5. This turns out to be sufficient, together with some moment bounds, to show strong convergence of the numerical scheme in Theorem 5.6. Our method of proof does not give rates for the strong convergence.

While strong convergence results for numerical schemes for SPDEs with globally Lipschitz coefficients, or at least some sort of linear growth condition, are abundant there are only few results on strong convergence of discretization schemes for SPDEs with superlinearly growing coefficients [1, 14, 15, 18, 20, 21, 24]. Furthermore, these papers dominantly establish strong convergence of various numerical schemes with no rate given (with a few exceptions), under some sort of global monotonicity assumption on the drift term which is not valid for the Cahn-Hilliard-Cook equation (1.1).

The analysis of numerical methods for SPDEs without a global monotonicity assumption is even less explored. For the Cahn-Hilliard-Cook equation (1.1) studied in the present paper, convergence (with rates) in probability is established for a finite difference scheme in [6]. In [16] strong convergence with rates is established for the spatial spectral Galerkin approximation (no time discretization) for (1.1) and the stochastic Burgers equation driven by trace class noise in spatial dimension $d=1$. The analysis is based on a general perturbation result and exponential integrability properties of the approximation process. Strong convergence of the finite element method without rate and without time discretization is proved in 22, 23 under stronger assumptions on the noise than in the present paper. There it is required that the operator composition $A^{\frac{\gamma}{2}} Q^{\frac{1}{2}}$ for $\gamma>1$ is Hilbert-Schmidt, while here we only require this with $\gamma=1$. Therefore, the present work can be viewed as the (non-trivial) extension of [22, 23] to a strongly convergent fully discrete scheme, still without a strong rate, but with improvements on the regularity requirement on the noise. Both here and in [22, 23] the strategy is based on proving a priori moment bounds with large exponents and in higher order norms using energy arguments and bootstrapping followed by a pathwise Gronwall argument in the mild solution setting. Finally, in connection to the Cahn-Hilliard-Cook equation we note that in [19, 25] the linearized Cahn-Hilliard-Cook equation is treated numerically which requires a significantly simpler analysis.

As further related work on numerical approximation of SPDEs without a global monotonicity assumption we mention the pathwise convergence of a spectral Galerkin method for the stochastic Burgers equation studied in [2, 3], while for the same equation convergence in probability is established in [26] for the Backward Euler method. The stochastic Navier-Stokes equation is considered in [5, 7], in particular, 
in [7] the authors obtain a result similar to our Theorem [5.5 (stated in a slightly different form). Finally, we mention the recent work [17, where strong convergence is proved, without rate, for a spectral nonlinearity-truncated accelerated exponential Euler-type approximation for the stochastic Kuramoto-Sivashinsky equation driven by space-time white noise in spatial dimension $d=1$, an equation rather similar in structure to the Cahn-Hilliard-Cook equation.

The paper is organized as follows. In Section 2 we collect some background material from stochastic and functional analysis and introduce the finite element method in Subsection 2.2. In Section 3 some known results on the existence, uniqueness and regularity on the solution of (1.1) are recalled. Section 4 contains moment bounds for the numerical solution, in particular, it contains the main technical result of the paper, Theorem 4.3. In Section 5 we prove a new error estimate for the derivative of the error in the spatial semidiscretization of the linear deterministic Cahn-Hilliard equation, (5.6) in Lemma 5.4 Then we proceed to prove a pathwise error bound in Theorem 5.5 and the main strong convergence result Theorem 5.6.

\section{Preliminaries}

2.1. Norms and operators. Throughout the paper we will use various norms for linear operators on a Hilbert space $H$ where the latter is endowed with inner product $\langle\cdot, \cdot\rangle$ and norm $\|\cdot\|$. We denote by $\mathcal{L}(H)$, the space of bounded linear operators on $H$ with the usual operator norm also denoted by $\|\cdot\|$. If for a selfadjoint positive semidefinite operator $T \in \mathcal{L}(H)$, the sum

$$
\operatorname{Tr} T:=\sum_{k=1}^{\infty}\left\langle T e_{k}, e_{k}\right\rangle<\infty
$$

for an orthonormal basis (ONB) $\left\{e_{k}\right\}_{k \in \mathbb{N}}$ of $H$, then we say that $T$ is trace class. In this case $\operatorname{Tr} T$, the trace of $T$, is independent of the choice of the ONB. If for an operator $T \in \mathcal{L}(H)$, the sum

$$
\|T\|_{\mathrm{HS}}^{2}:=\sum_{k=1}^{\infty}\left\|T e_{k}\right\|^{2}<\infty
$$

for an ONB $\left\{e_{k}\right\}_{k \in \mathbb{N}}$ of $H$, then we say that $T$ is Hilbert-Schmidt and call $\|T\|_{\text {HS }}$ the Hilbert-Schmidt norm of $T$. The Hilbert-Schmidt norm of $T$ is independent of the choice of the ONB. We have the following well-known properties of the trace and Hilbert-Schmidt norms, see, for example, [10, Appendix C],

$$
\begin{aligned}
& \|T\| \leq\|T\|_{\mathrm{HS}}, \quad\|T S\|_{\mathrm{HS}} \leq\|T\|_{\mathrm{HS}}\|S\|, \quad\|S T\|_{\mathrm{HS}} \leq\|S\|\|T\|_{\mathrm{HS}}, \\
& \operatorname{Tr} Q=\left\|Q^{\frac{1}{2}}\right\|_{\mathrm{HS}}^{2}=\|T\|_{\mathrm{HS}}^{2}=\left\|T^{*}\right\|_{\mathrm{HS}}^{2}, \quad \text { if } Q=T T^{*} .
\end{aligned}
$$

Next we introduce spaces and norms associated with the operator $A$, the negative of the Neumann Laplacian. Let $\mathcal{D} \subset \mathbb{R}^{d}, d=1,2,3$, be a bounded convex domain with polygonal boundary $\partial \mathcal{D}$. We denote by $\|\cdot\|_{L_{p}}$ the standard norm in $L_{p}(\mathcal{D})$. In particular, we define $H=L_{2}(\mathcal{D})$ with its standard inner product $\langle\cdot, \cdot\rangle$ and norm $\|\cdot\|$, and

$$
\dot{H}=\left\{v \in H: \int_{\mathcal{D}} v \mathrm{~d} x=0\right\} .
$$

Let $P: H \rightarrow \dot{H}$ define the orthogonal projector. Then $(I-P) v=|\mathcal{D}|^{-1} \int_{\mathcal{D}} v \mathrm{~d} x$ is the average of $v$. We also denote by $H^{k}(\mathcal{D})$ the standard Sobolev space. We define 
$A=-\Delta$, the negative of the Neumann Laplacian with domain of definition

$$
D(A)=\left\{v \in H^{2}(\mathcal{D}): \frac{\partial v}{\partial n}=0 \text { on } \partial \mathcal{D}\right\} .
$$

Then $A$ is a positive definite, selfadjoint, unbounded, linear operator on $\dot{H}$ with compact inverse. When extended to $H$ as $A v=A P v$ it has an orthonormal eigenbasis $\left\{\varphi_{j}\right\}_{j=0}^{\infty}$ with corresponding eigenvalues $\left\{\lambda_{j}\right\}_{j=0}^{\infty}$ such that

$$
0=\lambda_{0}<\lambda_{1} \leq \lambda_{2} \leq \cdots \leq \lambda_{j} \leq \cdots, \quad \lambda_{j} \rightarrow \infty
$$

see, for example, [11, Section 7.2]. The first eigenfunction is constant, $\varphi_{0}=|\mathcal{D}|^{-\frac{1}{2}}$.

We define

$$
\begin{aligned}
|v|_{\alpha} & =\left(\sum_{j=1}^{\infty} \lambda_{j}^{\alpha}\left|\left\langle v, \varphi_{j}\right\rangle\right|^{2}\right)^{\frac{1}{2}}, \quad\langle v, w\rangle_{\alpha}=\sum_{j=1}^{\infty} \lambda_{j}^{\alpha}\left\langle v, \varphi_{j}\right\rangle\left\langle w, \varphi_{j}\right\rangle, \quad \alpha \in \mathbb{R}, \\
\|v\|_{\alpha} & =\left(|v|_{\alpha}^{2}+\left|\left\langle v, \varphi_{0}\right\rangle\right|^{2}\right)^{\frac{1}{2}}, \quad \alpha \geq 0,
\end{aligned}
$$

and corresponding spaces, for $\alpha \geq 0$,

$$
\dot{H}^{\alpha}=D\left(A^{\frac{\alpha}{2}}\right)=\left\{v \in \dot{H}:|v|_{\alpha}<\infty\right\}, \quad H^{\alpha}=\left\{v \in H:\|v\|_{\alpha}<\infty\right\} .
$$

For negative order $-\alpha<0$ we define $\dot{H}^{-\alpha}$ by taking the closure of $\dot{H}$ with respect to $|\cdot|_{-\alpha}$. For integer order $\alpha=k=1,2$, the norm $\|\cdot\|_{k}$ is equivalent on $H^{k}$ to the standard Sobolev norm $\|\cdot\|_{H^{k}(\mathcal{D})}$. More precisely,

$$
\begin{aligned}
& \|v\|_{1}=\left(|v|_{1}^{2}+\left|\left\langle v, \varphi_{0}\right\rangle\right|^{2}\right)^{\frac{1}{2}}=\left(\|\nabla v\|^{2}+\left|\left\langle v, \varphi_{0}\right\rangle\right|^{2}\right)^{\frac{1}{2}}, \\
& \|v\|_{2}=\left(|v|_{2}^{2}+\left|\left\langle v, \varphi_{0}\right\rangle\right|^{2}\right)^{\frac{1}{2}}=\left(\|\Delta v\|^{2}+\left|\left\langle v, \varphi_{0}\right\rangle\right|^{2}\right)^{\frac{1}{2}}
\end{aligned}
$$

are equivalent to the standard norms $\|v\|_{H^{k}(\mathcal{D})}, k=1,2$, by the Poincaré inequality and the regularity estimate for the elliptic Neumann problem.

We recall the fact the operator $-A^{2}$ is the infinitesimal generator of an analytic semigroup $E(t)=\mathrm{e}^{-t A^{2}}$ on $H$,

$$
\begin{aligned}
E(t) v=\mathrm{e}^{-t A^{2}} v & =\sum_{j=0}^{\infty} \mathrm{e}^{-t \lambda_{j}^{2}}\left\langle v, \varphi_{j}\right\rangle \varphi_{j}=\sum_{j=1}^{\infty} \mathrm{e}^{-t \lambda_{j}^{2}}\left\langle v, \varphi_{j}\right\rangle \varphi_{j}+\left\langle v, \varphi_{0}\right\rangle \varphi_{0} \\
& =P \mathrm{e}^{-t A^{2}} v+(I-P) v .
\end{aligned}
$$

By expansion in the eigenbasis of $A$ and using Parseval's identity we easily obtain

$$
\left\|A^{\alpha} E(t) v\right\| \leq C t^{-\frac{\alpha}{2}}\|v\|, \quad v \in H, \alpha \geq 0 .
$$

and

$$
\left(\int_{0}^{t} s^{2 j}\left\|A^{2 j+1} E(s) v\right\|^{2} \mathrm{~d} s\right)^{1 / 2} \leq C\|v\|, \quad v \in H, j=0,1,2 \ldots .
$$

Here $C$ depends on $\alpha$ and $j$, respectively. 
2.2. The finite element method. Let $\left\{\mathcal{T}_{h}\right\}_{h>0}$ denote a family of regular triangulations of $\mathcal{D}$ with maximal mesh size $h$. Let $S_{h}$ be the space of continuous functions on $\mathcal{D}$, which are piecewise polynomials of degree $\leq 1$ with respect to $\mathcal{T}_{h}$. Hence, $S_{h} \subset H^{1}$. We also define $\dot{S}_{h}=P S_{h}$; that is,

$$
\dot{S}_{h}=\left\{v_{h} \in S_{h}: \int_{\mathcal{D}} v_{h} \mathrm{~d} x=0\right\} .
$$

The space $\dot{S}_{h}$ is introduced only for the purpose of theory but not for computation. Now we define the discrete Laplacian $-A_{h}: S_{h} \rightarrow S_{h}$ by

$$
\left\langle A_{h} v_{h}, w_{h}\right\rangle=\left\langle\nabla v_{h}, \nabla w_{h}\right\rangle, \quad \forall v_{h} \in S_{h}, w_{h} \in S_{h} .
$$

The operator $A_{h}$ is selfadjoint, positive definite on $\dot{S}_{h}$, positive semidefinite on $S_{h}$, and $A_{h}$ has an orthonormal eigenbasis $\left\{\varphi_{h, j}\right\}_{j=0}^{N_{h}}$ with corresponding eigenvalues $\left\{\lambda_{h, j}\right\}_{j=0}^{N_{h}}$. We have

$$
0=\lambda_{h, 0}<\lambda_{h, 1} \leq \cdots \leq \lambda_{h, j} \leq \cdots \leq \lambda_{h, N_{h}}
$$

and $\varphi_{h, 0}=\varphi_{0}=|\mathcal{D}|^{-\frac{1}{2}}$. Moreover, we define $E_{h}(t)=\mathrm{e}^{-t A_{h}^{2}}: S_{h} \rightarrow S_{h}$ by

$$
E_{h}(t) v_{h}=\mathrm{e}^{-t A_{h}^{2}} v_{h}=\sum_{j=0}^{N_{h}} \mathrm{e}^{-t \lambda_{h, j}}\left\langle v_{h}, \varphi_{h, j}\right\rangle \varphi_{h, j}
$$

and the orthogonal projector $P_{h}: H \rightarrow S_{h}$ by

$$
\left\langle P_{h} v, w_{h}\right\rangle=\left\langle v, w_{h}\right\rangle \quad \forall v \in H, w_{h} \in S_{h} .
$$

Clearly, $P_{h}: \dot{H} \rightarrow \dot{S}_{h}$ and

$$
E_{h}(t) P_{h} v=P E_{h}(t) P_{h} v+(I-P) v .
$$

Likewise, for its time discrete analog $R_{k, h}^{n}=\left(I+k A_{h}^{2}\right)^{-n}$, we have

$$
R_{k, h}^{n} P_{h} v=P R_{k, h}^{n} P_{h} v+(I-P) v .
$$

We have the discrete analogs of (2.6),

$$
\left\|A_{h}^{\alpha} E_{h}(t) P_{h} v\right\| \leq C t^{-\frac{\alpha}{2}}\|v\|, \quad\left\|A_{h}^{\alpha} R_{k, h}^{n} P_{h} v\right\| \leq C t_{n}^{-\frac{\alpha}{2}}\|v\|, \quad v \in H, \alpha \geq 0,
$$

where the constants $C$ depend on $\alpha$ but not on $h$ and $k$. Similarly to (2.6), these are proved by expansion in the eigenbasis of $A_{h}$ and Parseval's identity. For example, the first constant is $C=\left(\sup _{s \in[0, \infty)} s^{\alpha} \mathrm{e}^{-2 s}\right)^{\frac{1}{2}}$.

Finally, we define the Ritz projector $R_{h}: \dot{H}^{1} \rightarrow \dot{S}_{h}$ by

$$
\left\langle\nabla R_{h} v, \nabla w_{h}\right\rangle=\left\langle\nabla v, \nabla w_{h}\right\rangle, \quad \forall v \in \dot{H}^{1}, w_{h} \in \dot{S}_{h} .
$$

We extend it as $R_{h}: H^{1} \rightarrow S_{h}$ by

$$
R_{h} v=R_{h} P v+(I-P) v, \quad v \in H^{1} .
$$

We then have the following bound for $R_{h} v-v=\left(R_{h}-I\right) P v$ (cf. [27, Chapt. 1])

$$
\left\|\left(R_{h}-I\right) v\right\| \leq C h^{2}\|A v\|, \quad v \in \dot{H}^{2} .
$$

Finally, we define norms on $\dot{S}_{h}$, analogous to the norms $|\cdot|_{\alpha}$ on $\dot{H}^{\alpha}$ :

$$
\left|v_{h}\right|_{\alpha, h}=\left\|A_{h}^{\alpha / 2} v_{h}\right\|=\left(\sum_{j=1}^{N_{h}} \lambda_{j, h}^{\alpha}\left|\left\langle v, \varphi_{j, h}\right\rangle\right|^{2}\right)^{\frac{1}{2}}, \quad v_{h} \in \dot{S}_{h}, \alpha \in \mathbb{R} .
$$


The corresponding scalar products are denoted $\langle\cdot, \cdot\rangle_{\alpha, h}$. We note that

$$
\left|v_{h}\right|_{1}=\left\|A^{\frac{1}{2}} v_{h}\right\|=\left\|\nabla v_{h}\right\|=\left\|A_{h}^{\frac{1}{2}} v_{h}\right\|=\left|v_{h}\right|_{1, h}, \quad v_{h} \in \dot{S}_{h} .
$$

We assume that $P_{h}$ is bounded with respect to the $\dot{H}^{1}$ norm

$$
\left|P_{h} v\right|_{1} \leq C|v|_{1}, \quad v \in \dot{H}^{1} .
$$

This holds, for example, if the mesh family $\left\{\mathcal{T}_{h}\right\}_{h>0}$ is quasi-uniform. By combining this with (2.14), we obtain

$$
\left\|A_{h}^{1 / 2} P_{h} v\right\|=\left|P_{h} v\right|_{1} \leq C|v|_{1}=C\left\|A^{1 / 2} v\right\| .
$$

2.3. Useful inequalities. We will use the Burkholder-Davis-Gundy inequality for Itô-integrals of the form $\int_{0}^{t}\langle\eta(s), \mathrm{d} \tilde{W}(s)\rangle$, where $\eta$ is a predictable $H$-valued stochastic process and $\tilde{W}$ is a $\tilde{Q}$-Wiener process in $H$. For this kind of integral, the Burkholder-Davis-Gundy inequality, [10, Lemma 7.2], takes the form

$$
\mathbf{E} \sup _{t \in[0, T]}\left|\int_{0}^{t}\langle\eta(s), \mathrm{d} \tilde{W}(s)\rangle\right|^{p} \leq C \mathbf{E}\left(\int_{0}^{T}\left\|\tilde{Q}^{\frac{1}{2}} \eta(s)\right\|^{2} \mathrm{~d} s\right)^{\frac{p}{2}}, \quad p \geq 2,
$$

where $C$ depends on $p$.

Also, if $Y$ is an $H$-valued centered Gaussian random variable with covariance operator $\tilde{Q}$, then, by [10, Corollary 2.17], we can bound its $p$-th moments via its covariance operator as

$$
\mathbf{E}\|Y\|^{2 p} \leq C\left(\mathbf{E}\|Y\|^{2}\right)^{p}=C(\operatorname{Tr} \tilde{Q})^{p}=C\left\|\tilde{Q}^{\frac{1}{2}}\right\|_{\mathrm{HS}}^{2 p}, \quad p \geq 1,
$$

where $C$ depends on $p$. In particular, for an Itô integral $Y=\int_{s}^{t} R \mathrm{~d} W(r)=$ $R(W(t)-W(s))$, where $R$ is a constant, possibly unbounded, operator on $H$ and $W$ is a $Q$-Wiener process, the inequality (2.18) reads

$$
\mathbf{E}\left\|\int_{s}^{t} R \mathrm{~d} W(r)\right\|^{2 p} \leq C(t-s)^{p}\left\|R Q^{1 / 2}\right\|_{\mathrm{HS}}^{2 p} .
$$

If $p=1$, the inequality in (2.19) becomes an equality with $C=1$. The inequality

$$
\left|\sum_{j=K}^{M} a_{j}\right|^{p} \leq|M-K+1|^{p-1} \sum_{j=K}^{M}\left|a_{j}\right|^{p}, \quad p \geq 1,
$$

will be frequently utilized; it is a direct consequence of Hölder's inequality.

\section{EXISTENCE, UNIQUENESS AND REGULARITY}

Existence, uniqueness, and regularity of weak solutions to (1.1) has been studied in 9 with some minor improvements in 22. Note that here we assume that $X_{0}$ is deterministic and that $X_{0} \in \dot{H}$, so that $X(t) \in \dot{H}$. We summarize the results:

Theorem 3.1. If $\left\|A^{\frac{1}{2}} Q^{\frac{1}{2}}\right\|_{\mathrm{HS}}<\infty,\left|X_{0}\right|_{1}<\infty$, and $T<\infty$, then there is a unique weak solution $X$ of (1.1); that is, an adapted $H$-valued process $X$, which is continuous almost surely and satisfies the equation

$$
\langle X(t), v\rangle-\left\langle X_{0}, v\right\rangle+\int_{0}^{t}\left(\left\langle X(s), A^{2} v\right\rangle+\langle f(X(s)), A v\rangle\right) \mathrm{d} s=\langle W(t), v\rangle
$$


almost surely for all $v \in \dot{H}^{4}=D\left(A^{2}\right), t \in[0, T]$. Furthermore, there is $C>0$ such that

$$
\mathbf{E} \sup _{t \in[0, T]}|X(t)|_{1}^{2}+\mathbf{E} \sup _{t \in[0, T]}\|X(t)\|_{L_{4}}^{4} \leq C .
$$

In addition, $X$ is also a mild solution; that is, it satisfies the equation

$$
X(t)=E(t) X_{0}-\int_{0}^{t} E(t-s) A f(X(s)) \mathrm{d} s+\int_{0}^{t} E(t-s) \mathrm{d} W(s),
$$

almost surely.

We also have pathwise Hölder regularity in time:

Proposition 3.2. Let $\left\|A^{\frac{1}{2}} Q^{\frac{1}{2}}\right\|_{\mathrm{HS}}<\infty$ and $\left|X_{0}\right|_{1}<\infty$. Then, for all $\gamma \in\left[0, \frac{1}{2}\right)$, there is an almost surely finite nonnegative random variable $K$ such that, almost surely,

$$
\sup _{t \neq s \in[0, T]} \frac{\|X(t)-X(s)\|}{|t-s|^{\gamma}} \leq K .
$$

We omit the proof as it is analogous to the proof of [20, Proposition 3.2].

\section{Moment Bounds For the Discrete solution}

We start by proving a preliminary moment bound which will be used later on in a bootstrapping argument. Throughout the proofs, $C$ denotes a generic nonnegative constant that is independent of the discretization parameters $h$ and $k$ and may change from line to line.

We recall our assumption that $X_{0} \in \dot{H}$, so that $X_{h}^{0}=P_{h} X_{0} \in \dot{S}_{h}$ and hence $X_{h}^{j} \in \dot{S}_{h}$ for $0 \leq j \leq N$.

Lemma 4.1. Let $p \geq 1$. If $\left\|A_{h}^{-1 / 2} P_{h} Q^{1 / 2}\right\|_{\mathrm{HS}} \leq K$ and $\left|X_{h}^{0}\right|_{-1, h} \leq L$ for all $h>0$, then there exists $C>0$ depending on $p, T, K, L$, such that, for all $h, k>0$,

$$
\begin{aligned}
& \mathbf{E}\left(\sup _{1 \leq j \leq N}\left|X_{h}^{j}\right|_{-1, h}^{2 p}\right) \leq C, \\
& \mathbf{E}\left(\sum_{j=1}^{N}\left(\left|X_{h}^{j}-X_{h}^{j-1}\right|_{-1, h}^{2}+k\left|X_{h}^{j}\right|_{1}^{2}\right)\right)^{p} \leq C .
\end{aligned}
$$

Proof. Since $X_{h}^{j} \in \dot{S}_{h}$, we may multiply by $A_{h}^{-1} X_{h}^{j}$ in (1.6) to get

$$
\begin{aligned}
& \frac{1}{2}\left(\left|X_{h}^{j}\right|_{-1, h}^{2}-\left|X_{h}^{j-1}\right|_{-1, h}^{2}+\left|X_{h}^{j}-X_{h}^{j-1}\right|_{-1, h}^{2}\right)+k\left|X_{h}^{j}\right|_{1}^{2}+k\left\langle f\left(X_{h}^{j}\right), X_{h}^{j}\right\rangle \\
& =\left\langle P_{h} \Delta W^{j}, A_{h}^{-1} X_{h}^{j}\right\rangle,
\end{aligned}
$$

where we have used the selfadjointness of $A_{h}$, (2.14), and the identity

$$
\langle X-Y, X\rangle_{-1, h}=\frac{1}{2}\left(|X|_{-1, h}^{2}-|Y|_{-1, h}^{2}+|X-Y|_{-1, h}^{2}\right) .
$$

Next, the dissipativity inequality (1.3) yields

$$
\begin{aligned}
& \frac{1}{2}\left(\left|X_{h}^{j}\right|_{-1, h}^{2}-\left|X_{h}^{j-1}\right|_{-1, h}^{2}+\left|X_{h}^{j}-X_{h}^{j-1}\right|_{-1, h}^{2}\right)+k\left|X_{h}^{j}\right|_{1}^{2} \\
& \quad \leq C_{0} k+\left\langle P_{h} \Delta W^{j}, X_{h}^{j}-X_{h}^{j-1}\right\rangle_{-1, h}+\left\langle P_{h} \Delta W^{j}, X_{h}^{j-1}\right\rangle_{-1, h} .
\end{aligned}
$$


Furthermore, $\left\langle P_{h} \Delta W^{j}, X_{h}^{j}-X_{h}^{j-1}\right\rangle_{-1, h} \leq C_{\epsilon}\left|P_{h} \Delta W^{j}\right|_{-1, h}^{2}+\epsilon\left|X_{h}^{j}-X_{h}^{j-1}\right|_{-1, h}^{2}$. Hence,

$$
\begin{gathered}
\frac{1}{2}\left(\left|X_{h}^{j}\right|_{-1, h}^{2}-\left|X_{h}^{j-1}\right|_{-1, h}^{2}\right)+c\left|X_{h}^{j}-X_{h}^{j-1}\right|_{-1, h}^{2}+k\left|X_{h}^{j}\right|_{1}^{2} \\
\leq C\left(k+\left|P_{h} \Delta W^{j}\right|_{-1, h}^{2}+\left\langle P_{h} \Delta W^{j}, X_{h}^{j-1}\right\rangle_{-1, h}\right) .
\end{gathered}
$$

Summing with respect to $j$ in (4.4), thus yields

$$
\begin{gathered}
\left|X_{h}^{n}\right|_{-1, h}^{2}+\sum_{j=1}^{n}\left(\left|X_{h}^{j}-X_{h}^{j-1}\right|_{-1, h}^{2}+k\left|X_{h}^{j}\right|_{1}^{2}\right) \leq C\left(T+\left|X_{h}^{0}\right|_{-1, h}^{2}\right. \\
\left.+\sum_{j=1}^{n}\left|P_{h} \Delta W^{j}\right|_{-1, h}^{2}+\sum_{j=1}^{n}\left\langle P_{h} \Delta W^{j}, X_{h}^{j-1}\right\rangle_{-1, h}\right) .
\end{gathered}
$$

We drop the sum on the left, take the $p^{\prime}$ th power and the supremum with respect to $n$, and then the expectation, using also (2.20) repeatedly, to get

$$
\begin{aligned}
& \mathbf{E} \sup _{1 \leq n \leq N}\left|X_{h}^{n}\right|_{-1, h}^{2 p} \leq \mathbf{E} \sup _{1 \leq n \leq N} C\left\{T+\left|X_{h}^{0}\right|_{-1, h}^{2}\right. \\
&\left.\quad+\sum_{j=1}^{n}\left|P_{h} \Delta W^{j}\right|_{-1, h}^{2}+\sum_{j=1}^{n}\left\langle P_{h} \Delta W^{j}, X_{h}^{j-1}\right\rangle_{-1, h}\right\}^{p} \\
& \leq C \mathbf{E}\left\{T^{p}+\left|X_{h}^{0}\right|_{-1, h}^{2 p}\right. \\
&\left.+\left(\sum_{j=1}^{N}\left|P_{h} \Delta W^{j}\right|_{-1, h}^{2}\right)^{p}+\sup _{1 \leq n \leq N}\left|\sum_{j=1}^{n}\left\langle P_{h} \Delta W^{j}, X_{h}^{j-1}\right\rangle_{-1, h}\right|^{p}\right\} \\
& \leq C\left\{T^{p}+\left|X_{h}^{0}\right|_{-1, h}^{2 p}\right. \\
&\left.+\mathbf{E}\left(\sum_{j=1}^{N}\left|P_{h} \Delta W^{j}\right|_{-1, h}^{2}\right)^{p}+\mathbf{E} \sup _{1 \leq n \leq N}\left|\sum_{j=1}^{n}\left\langle P_{h} \Delta W^{j}, X_{h}^{j-1}\right\rangle_{-1, h}\right|^{p}\right\} .
\end{aligned}
$$

By (2.18), we have

$$
\begin{aligned}
& \mathbf{E}\left(\sum_{j=1}^{N}\left|P_{h} \Delta W^{j}\right|_{-1, h}^{2}\right)^{p} \leq C N^{p-1} \sum_{j=1}^{N} \mathbf{E}\left|P_{h} \Delta W^{j}\right|_{-1, h}^{2 p} \\
& \quad \leq C N^{p-1} \sum_{j=1}^{N} k^{p}\left\|A_{h}^{-1 / 2} P_{h} Q^{1 / 2}\right\|_{\mathrm{HS}}^{2 p} \leq C T^{p}\left\|A_{h}^{-1 / 2} P_{h} Q^{1 / 2}\right\|_{\mathrm{HS}}^{2 p} \leq C .
\end{aligned}
$$


Moreover, by Cauchy's inequality and (2.17),

$$
\begin{aligned}
& \left|\sum_{j=1}^{n}\left\langle P_{h} \Delta W^{j}, X_{h}^{j-1}\right\rangle_{-1, h}\right|^{p}=\mathbf{E} \sup _{1 \leq n \leq N}\left|\sum_{j=1}^{n}\left\langle\Delta W^{j}, A_{h}^{-1} X_{h}^{j-1}\right\rangle\right|^{p} \\
& \quad \leq C \mathbf{E}\left(\sum_{j=1}^{N} k\left\|Q^{1 / 2} A_{h}^{-1} X_{h}^{j-1}\right\|^{2}\right)^{p / 2} \\
& \quad \leq C\left\{1+\mathbf{E}\left(\sum_{j=1}^{N} k\left\|Q^{1 / 2} A_{h}^{-1} X_{h}^{j-1}\right\|^{2}\right)^{p}\right\} \\
& \quad \leq C\left\{1+(N k)^{p-1} \sum_{j=1}^{N} k \mathbf{E}\left\|Q^{1 / 2} A_{h}^{-1} P_{h} X_{h}^{j-1}\right\|^{2 p}\right\} \\
& \quad \leq C\left\{1+(N k)^{p-1} \sum_{j=1}^{N} k\left\|Q^{1 / 2} A_{h}^{-1 / 2} P_{h}\right\|^{2 p} \mathbf{E}\left|X_{h}^{j-1}\right|_{-1, h}^{2 p}\right\} \\
& \quad=C\left\{1+(N k)^{p-1} \sum_{j=1}^{N} k\left\|A_{h}^{-1 / 2} P_{h} Q^{1 / 2}\right\|^{2 p} \mathbf{E}\left|X_{h}^{j-1}\right|_{-1, h}^{2 p}\right\}
\end{aligned}
$$

As $N k=T,\left\|A_{h}^{-1 / 2} P_{h} Q^{1 / 2}\right\| \leq\left\|A_{h}^{-1 / 2} P_{h} Q^{1 / 2}\right\|_{\mathrm{HS}} \leq K$, and $\left|X_{h}^{0}\right|_{-1, h} \leq L$, by inserting (4.7) and (4.8) into (4.6), we see that

$$
\begin{gathered}
\mathbf{E} \sup _{0 \leq n \leq N}\left|X_{h}^{n}\right|_{-1, h}^{2 p} \leq C(p, T, K, L)\left(1+\sum_{j=0}^{N-1} k \mathbf{E}\left|X_{h}^{j}\right|_{-1, h}^{2 p}\right) \\
=C(p, T, K, L)\left(1+\sum_{n=0}^{N-1} k \mathbf{E} \sup _{0 \leq j \leq n}\left|X_{h}^{j}\right|_{-1, h}^{2 p}\right)
\end{gathered}
$$

By induction, (4.9) shows that the quantity $\mathbf{E} \sup _{0 \leq j \leq n}\left|X_{h}^{j}\right|_{-1, h}^{2 p}$ is finite for all $n=1, \ldots, N$ and hence the inequality (4.1) follows from Gronwall's lemma. Having this result at hand one may return to (4.5) to prove (4.2) by a similar procedure but without the Gronwall argument at the end.

In the sequel, in many places the quantity

$$
Y_{h}^{j}:=A_{h} X_{h}^{j}+P_{h} f\left(X_{h}^{j}\right)
$$

plays a crucial role. It can be regarded as the discrete version of the "chemical potential" $Y=A X+f$. With this notation, the scheme (1.6) can be rewritten as

$$
X_{h}^{j}-X_{h}^{j-1}+k A_{h} Y_{h}^{j}=P_{h} \Delta W^{j}, j=1,2, \ldots, N ; \quad X_{h}^{0}=P_{h} X_{0} .
$$

We continue to prove a stronger moment bound. Note that it is not "closed", because $Y_{h}^{j}$ remains on the right hand side.

Lemma 4.2. Suppose that $\left\|A^{1 / 2} Q^{1 / 2}\right\|_{\mathrm{HS}} \leq K$ and $\left|X_{h}^{0}\right|_{-1, h} \leq L$ for all $h>0$. Then, for every $\epsilon, \delta>0$ and $p \geq 1$, there are $C_{1}>0$ depending on $T, \epsilon, \delta, p, K$ and 
$L$, and $C_{2}>0$ depending on $T$ and $p$ such that for all $h, k>0$,

$$
\mathbf{E}\left(\sup _{1 \leq j \leq N}\left\|X_{h}^{j}\right\|^{2 p}\right)+\mathbf{E}\left(\sum_{j=1}^{N}\left\|X_{h}^{j}-X_{h}^{j-1}\right\|^{2}\right)^{p} \leq C_{1}+C_{2} \delta \mathbf{E}\left(\sum_{j=1}^{N} k\left|Y_{h}^{j}\right|_{1}^{2}\right)^{\frac{1+\epsilon}{2} p} .
$$

Proof. By taking inner products with $X_{h}^{j} \in \dot{S}_{h}$ in (4.11) we get

$$
\frac{1}{2}\left(\left\|X_{h}^{j}\right\|^{2}-\left\|X_{h}^{j-1}\right\|^{2}+\left\|X_{h}^{j}-X_{h}^{j-1}\right\|^{2}\right)+k\left\langle Y_{h}^{j}, X_{h}^{j}\right\rangle_{1}=\left\langle\Delta W^{j}, X_{h}^{j}\right\rangle,
$$

where we recall that $\langle x, y\rangle_{1}=\langle\nabla x, \nabla y\rangle$. Summing with respect to $j$, using analogous arguments as in the previous proof, thus yields for $1 \leq n \leq N$

$$
\begin{aligned}
\left\|X_{h}^{n}\right\|^{2}+\sum_{j=1}^{n}\left\|X_{h}^{j}-X_{h}^{j-1}\right\|^{2} \leq & C\left(\left\|X_{h}^{0}\right\|^{2}+\sum_{j=1}^{n} k\left|\left\langle Y_{h}^{j}, X_{h}^{j}\right\rangle_{1}\right|\right. \\
& \left.+\sum_{j=1}^{n}\left\|\Delta W^{j}\right\|^{2}+\sum_{j=1}^{n}\left\langle\Delta W^{j}, X_{h}^{j-1}\right\rangle\right) .
\end{aligned}
$$

Therefore,

$$
\begin{aligned}
\mathbf{E} \sup _{1 \leq j \leq N}\left\|X_{h}^{j}\right\|^{2 p}+\mathbf{E}\left(\sum_{j=1}^{N}\left\|X_{h}^{j}-X_{h}^{j-1}\right\|^{2}\right)^{p} \\
\leq C\left\{\left\|X_{h}^{0}\right\|^{2 p}+\mathbf{E}\left(\sum_{j=1}^{N} k\left|\left\langle Y_{h}^{j}, X_{h}^{j}\right\rangle_{1}\right|\right)^{p}\right. \\
\left.\quad+\mathbf{E}\left(\sum_{j=1}^{N}\left\|\Delta W^{j}\right\|^{2}\right)^{p}+\mathbf{E} \sup _{1 \leq n \leq N}\left|\sum_{j=1}^{n}\left\langle A_{h}^{1 / 2} P_{h} \Delta W^{j}, A_{h}^{-1 / 2} X_{h}^{j-1}\right\rangle\right|^{p}\right\} .
\end{aligned}
$$

The next to last term can be bounded, similarly to (4.7) in the previous proof, by $C T^{p}\left\|Q^{1 / 2}\right\|_{\mathrm{HS}}^{2 p}$. Using the (4.1) and a calculation similar to (4.8) we obtain

$$
\begin{aligned}
\mathbf{E} \sup _{1 \leq n \leq N}\left|\sum_{j=1}^{n}\left\langle A_{h}^{1 / 2} P_{h} \Delta W^{j}, A_{h}^{-1 / 2} X_{h}^{j-1}\right\rangle\right|^{p} & \leq C\left(1+T^{p}\left\|A_{h}^{1 / 2} P_{h} Q^{1 / 2}\right\|^{2 p}\right) \\
& \leq C\left(1+T^{p}\left\|A^{1 / 2} Q^{1 / 2}\right\|^{2 p}\right)
\end{aligned}
$$

where we also used (2.16). Finally,

$$
\begin{aligned}
& \mathbf{E}\left(\sum_{j=1}^{N} k\left|\left\langle Y_{h}^{j}, X_{h}^{j}\right\rangle_{1}\right|\right)^{p} \leq \mathbf{E}\left(\sum_{j=1}^{N} k\left|Y_{h}^{j}\right|_{1}\left|X_{h}^{j}\right|_{1}\right)^{p} \\
& \quad \leq \mathbf{E}\left\{\left(\sum_{j=1}^{N} k\left|Y_{h}^{j}\right|_{1}^{2}\right)^{p / 2}\left(\sum_{j=1}^{N} k\left|X_{h}^{j}\right|_{1}^{2}\right)^{p / 2}\right\} \\
& \quad \leq \mathbf{E}\left\{\delta\left(\sum_{j=1}^{N} k\left|Y_{h}^{j}\right|_{1}^{2}\right)^{\frac{1+\epsilon}{2} p}+C_{\epsilon, \delta}\left(\sum_{j=1}^{N} k\left|X_{h}^{j}\right|_{1}^{2}\right)^{\frac{1+\epsilon}{2 \epsilon} p}\right\},
\end{aligned}
$$


and the proof is complete in view of (4.2) by noting that

$$
\begin{aligned}
\left\|A_{h}^{-1 / 2} P_{h} Q^{1 / 2}\right\|_{\mathrm{HS}} & =\left\|A_{h}^{-1 / 2} P_{h} A^{-1 / 2} A^{1 / 2} Q^{1 / 2}\right\|_{\mathrm{HS}} \\
& \leq\left\|A_{h}^{-1 / 2} P_{h} A^{-1 / 2} P\right\|\left\|A^{1 / 2} Q^{1 / 2}\right\|_{\mathrm{HS}} \leq C\left\|A^{1 / 2} Q^{1 / 2}\right\|_{\mathrm{HS}} .
\end{aligned}
$$

We next prove the main stability result of the paper.

It is well known that, for the deterministic Cahn-Hilliard equation,

$$
\dot{u}+A v=0, t>0 ; \quad v=A u+f(u),
$$

the functional

$$
J(u):=\frac{1}{2}|u|_{1}^{2}+\mathcal{F}(u), \quad \text { where } \mathcal{F}(u)=\int_{\mathcal{D}} F(u) \mathrm{d} x,
$$

is a Ljapunov functional, that is, $J(u(t)) \leq J\left(u_{0}\right), t \geq 0$. This leads to a uniform bound for $|u(t)|_{1}^{2}+\|u(t)\|_{L_{4}}^{4}$. The proof proceeds by multiplication of the equation by the chemical potential $v$ and noting that $J^{\prime}(u)=A u+f(u)=v$ and $\langle\dot{u}, v\rangle=$ $\left\langle\dot{u}, J^{\prime}(u)\right\rangle=\frac{\mathrm{d}}{\mathrm{d} t} J(u)$. Thus, $\frac{\mathrm{d}}{\mathrm{d} t} J(u)+|v|_{1}^{2}=0$ and

$$
J(u(T))+\int_{0}^{T}|v(t)|_{1}^{2} \mathrm{~d} t=J\left(u_{0}\right)
$$

which is the desired result.

This was imitated for the spatially semidiscrete Cahn-Hilliard-Cook equation in 22 by applying the Itô formula to $J\left(X_{h}(t)\right)$. For the fully discrete equation (1.6) we do not have an Itô formula, so we must use a more direct imitation of the above calculation in the proof of the following theorem, which contains our main moment bounds.

In the proof we denote by $P_{\alpha}(x), x=\left(x_{1}, \ldots, x_{m}\right), x_{i} \geq 0$, any nonnegative quantity such that

$$
P_{\alpha}(x) \leq C\left(1+\sum_{i=1}^{m} x_{i}^{\alpha}\right) .
$$

Theorem 4.3. Let $p \geq 1$. If $\left\|A^{1 / 2} Q^{1 / 2}\right\|_{\mathrm{HS}} \leq K$ and

$$
\left|X_{h}^{0}\right|_{-1, h}+J\left(X_{h}^{0}\right)+\left|Y_{h}^{0}\right|_{1} \leq L \text { for all } h>0,
$$

then there exist $C, k_{0}>0$, depending on $p, K, L$, and $T$, such that for all $h>0$ and $0<k<k_{0}$,

$$
\mathbf{E} \sup _{1 \leq j \leq N} J\left(X_{h}^{j}\right)^{p}+\mathbf{E}\left(\sum_{j=1}^{N} k\left|Y_{h}^{j}\right|_{1}^{2}\right)^{p} \leq C .
$$

Proof. Following the procedure from the deterministic case, we multiply (4.11) by the discrete chemical potential $Y_{h}^{j}=A_{h} X_{h}^{j}+P_{h} f\left(X_{h}^{j}\right)$ :

$$
\left\langle Y_{h}^{j}, \Delta X_{h}^{j}\right\rangle+k\left|Y_{h}^{j}\right|_{1}^{1}=\left\langle Y_{h}^{j}, P_{h} \Delta W^{j}\right\rangle,
$$

where $\Delta X_{h}^{j}=X_{h}^{j}-X_{h}^{j-1}$. From (1.4) it follows that, for $X, Z \in \dot{H}$,

$$
\mathcal{F}(X)-\mathcal{F}(Z) \leq\langle f(X), X-Z\rangle+\frac{1}{2} c_{1}^{2}\|X-Z\|^{2} .
$$


Hence,

$$
\left\langle P_{h} f\left(X_{h}^{j}\right), \Delta X_{h}^{j}\right\rangle=\left\langle f\left(X_{h}^{j}\right), \Delta X_{h}^{j}\right\rangle \geq \mathcal{F}\left(X_{h}^{j}\right)-\mathcal{F}\left(X_{h}^{j-1}\right)-\frac{1}{2} c_{1}^{2}\left\|\Delta X_{h}^{j}\right\|^{2} .
$$

As in 4.3) we have

$$
\left\langle A_{h} X_{h}^{j}, \Delta X_{h}^{j}\right\rangle=\left\langle X_{h}^{j}, \Delta X_{h}^{j}\right\rangle_{1}=\frac{1}{2}\left(\left|X_{h}^{j}\right|_{1}^{2}-\left|X_{h}^{j-1}\right|_{1}^{2}+\left|\Delta X_{h}^{j}\right|_{1}^{2}\right) .
$$

By adding the latter two relations, we obtain

$$
\begin{aligned}
\left\langle Y_{h}^{j}, \Delta X_{h}^{j}\right\rangle & =\left\langle A_{h} X_{h}^{j}+P_{h} f\left(X_{h}^{j}\right), \Delta X_{h}^{j}\right\rangle \\
& \geq J\left(X_{h}^{j}\right)-J\left(X_{h}^{j-1}\right)+\frac{1}{2}\left|\Delta X_{h}^{j}\right|_{1}^{2}+\frac{1}{2} c_{1}^{2}\left\|\Delta X_{h}^{j}\right\|^{2} .
\end{aligned}
$$

This is the discrete analog of $\langle v, \dot{u}\rangle=\frac{\mathrm{d}}{\mathrm{d} t} J(u)$. By (4.11), we now have

$$
J\left(X_{h}^{j}\right)-J\left(X_{h}^{j-1}\right)+\frac{1}{2}\left|\Delta X_{h}^{j}\right|_{1}^{2}+k\left|Y_{h}^{j}\right|_{1}^{1} \leq\left\langle Y_{h}^{j}, P_{h} \Delta W^{j}\right\rangle-\frac{1}{2} c_{1}^{2}\left\|\Delta X_{h}^{j}\right\|^{2} .
$$

The remaining challenge is to deal with the term

$$
\left\langle Y_{h}^{j}, P_{h} \Delta W^{j}\right\rangle=\left\langle Y_{h}^{j-1}, P_{h} \Delta W^{j}\right\rangle+\left\langle Y_{h}^{j}-Y_{h}^{j-1}, P_{h} \Delta W^{j}\right\rangle .
$$

We begin by

$$
\left\langle Y_{h}^{j}-Y_{h}^{j-1}, P_{h} \Delta W^{j}\right\rangle=\left\langle A_{h} \Delta X_{h}^{j}, P_{h} \Delta W^{j}\right\rangle+\left\langle f\left(X_{h}^{j}\right)-f\left(X_{h}^{j-1}\right), P_{h} \Delta W^{j}\right\rangle .
$$

Here, by (2.16),

$$
\left\langle A_{h} \Delta X_{h}^{j}, P_{h} \Delta W^{j}\right\rangle \leq \epsilon\left|\Delta X_{h}^{j}\right|_{1}^{2}+C_{\epsilon}\left|\Delta W^{j}\right|_{1}^{2}
$$

and, as $P_{h} \Delta W^{j} \in \dot{S}_{h} \subset \dot{H}$,

$$
\begin{aligned}
& \left|\left\langle f\left(X_{h}^{j}\right)-f\left(X_{h}^{j-1}\right), P_{h} \Delta W^{j}\right\rangle\right|=\left|\left\langle P\left(f\left(X_{h}^{j}\right)-f\left(X_{h}^{j-1}\right)\right), P_{h} \Delta W^{j}\right\rangle\right| \\
& \quad=\left|\left\langle A_{h}^{-1 / 2} P_{h} P\left(f\left(X_{h}^{j}\right)-f\left(X_{h}^{j-1}\right)\right), A_{h}^{1 / 2} P_{h} \Delta W^{j}\right\rangle\right| \\
& \quad \leq\left\|A_{h}^{-1 / 2} P_{h} P\left(f\left(X_{h}^{j}\right)-f\left(X_{h}^{j-1}\right)\right)\right\|\left|P_{h} \Delta W^{j}\right|_{1} \\
& \quad \leq C\left\|A_{h}^{-1 / 2} P_{h} P\left(f\left(X_{h}^{j}\right)-f\left(X_{h}^{j-1}\right)\right)\right\|\left|\Delta W^{j}\right|_{1} .
\end{aligned}
$$

By using Hölder's and Sobolev's inequalities $(d \leq 3)$ we show

$$
\left\|A_{h}^{-1 / 2} P_{h} P f\right\|=\sup _{v_{h} \in \dot{S}_{h}} \frac{\left\langle f, v_{h}\right\rangle}{\left|v_{h}\right|_{1}} \leq \sup _{v_{h} \in \dot{S}_{h}} \frac{\|f\|_{L_{6 / 5}(\mathcal{D})}\left\|v_{h}\right\|_{L_{6}(\mathcal{D})}}{\left|v_{h}\right|_{1}} \leq C\|f\|_{L_{6 / 5}(\mathcal{D})} .
$$

Therefore, (1.5) implies

$$
\begin{aligned}
& \| A_{h}^{-1 / 2} P_{h} P\left(f\left(X_{h}^{j}\right)-f\left(X_{h}^{j-1}\right)\|\leq C\| f\left(X_{h}^{j}\right)-f\left(X_{h}^{j-1}\right) \|_{L_{6 / 5}(\mathcal{D})}\right. \\
& \quad \leq C\left(\int_{\mathcal{D}}\left|X_{h}^{j}-X_{h}^{j-1}\right|^{6 / 5}\left(1+\left(X_{h}^{j}\right)^{2}+\left(X_{h}^{j-1}\right)^{2}\right)^{6 / 5} \mathrm{~d} \xi\right)^{5 / 6} \\
& \quad \leq C\left(\int_{\mathcal{D}}\left|X_{h}^{j}-X_{h}^{j-1}\right|^{6} \mathrm{~d} \xi\right)^{1 / 6}\left(\int_{\mathcal{D}}\left(1+\left(X_{h}^{j}\right)^{2}+\left(X_{h}^{j-1}\right)^{2}\right)^{3 / 2} \mathrm{~d} \xi\right)^{2 / 3} \\
& \quad \leq C\left\|X_{h}^{j}-X_{h}^{j-1}\right\|_{L_{6}(\mathcal{D})}\left(1+\left\|X_{h}^{j}\right\|_{L_{3}(\mathcal{D})}^{2}+\left\|X_{h}^{j-1}\right\|_{L_{3}(\mathcal{D})}^{2}\right) .
\end{aligned}
$$

Further, with $p<q<r$ and $\lambda=\frac{p}{q} \frac{r-q}{r-p}$, we have

$$
\|X\|_{L_{q}(\mathcal{D})} \leq\|X\|_{L_{p}(\mathcal{D})}^{\lambda}\|X\|_{L_{r}(\mathcal{D})}^{1-\lambda},
$$


see [13, Proposition 6.10]. We take $p=2, q=3, r=4$, and hence $\lambda=\frac{1}{3}$, to conclude that

$$
\left\|X_{h}^{j}\right\|_{L_{3}(\mathcal{D})}^{2} \leq\left\|X_{h}^{j}\right\|_{L_{2}(\mathcal{D})}^{2 / 3}\left\|X_{h}^{j}\right\|_{L_{4}(\mathcal{D})}^{4 / 3}
$$

Thus, from (4.17), (4.18), (4.19), and since by Sobolev's inequality we have $\| X_{h}^{j}-$ $X_{h}^{j-1} \|_{L_{6}(\mathcal{D})} \leq C\left|X_{h}^{j}-X_{h}^{j-1}\right|_{1}$, it follows that

$$
\begin{aligned}
\left|\left\langle f\left(X_{h}^{j}\right)-f\left(X_{h}^{j-1}\right), P_{h} \Delta W^{j}\right\rangle\right| \leq C\left|\Delta W^{j}\right|_{1}\left|X_{h}^{j}-X_{h}^{j-1}\right|_{1} \\
\quad \times P_{2 / 3}\left(\left\|X_{h}^{j}\right\|_{L_{2}(\mathcal{D})},\left\|X_{h}^{j-1}\right\|_{L_{2}(\mathcal{D})}\right) P_{4 / 3}\left(\left\|X_{h}^{j}\right\|_{L_{4}(\mathcal{D})},\left\|X_{h}^{j-1}\right\|_{L_{4}(\mathcal{D})}\right) \\
\leq \epsilon\left|X_{h}^{j}-X_{h}^{j-1}\right|_{1}^{2}+C\left|\Delta W^{j}\right|_{1}^{2} P_{4 / 3}\left(\left\|X_{h}^{j}\right\|_{L_{2}(\mathcal{D})},\left\|X_{h}^{j-1}\right\|_{L_{2}(\mathcal{D})}\right) \\
\quad \times P_{8 / 3}\left(\left\|X_{h}^{j}\right\|_{L_{4}(\mathcal{D})},\left\|X_{h}^{j-1}\right\|_{L_{4}(\mathcal{D})}\right)
\end{aligned}
$$

where we used the notation $P_{\alpha}$ from (4.13). This means that we have bounded the Lipschitz constant in (4.18) by powers of $\left\|X_{h}^{j}\right\|_{L_{q}(\mathcal{D})}, q=2$, 4 , which we shall be able to control. It will be important that the exponent on $\left\|X_{h}^{j}\right\|_{L_{4}(\mathcal{D})}$, is strictly less than 4 so that it can be controled in terms of $\mathcal{F}\left(X_{h}^{j}\right)$. Therefore we cannot simplify by multiplying the polynomials together. The exponent on $\left\|X_{h}^{j}\right\|_{L_{2}(\mathcal{D})}$ can be arbitrarily large because of Lemma 4.2

Thus, with $0<\epsilon<\frac{1}{4}$ we get, after inserting (4.16) and (4.20) into (4.15) and rearranging, that

$$
\begin{aligned}
& J\left(X_{h}^{j}\right)-J\left(X_{h}^{j-1}\right)+c\left|X_{h}^{j}-X_{h}^{j-1}\right|_{1}^{2}+k\left|Y_{h}^{j}\right|_{1}^{2} \\
& \leq C\left|\Delta W^{j}\right|_{1}^{2} P_{4 / 3}\left(\left\|X_{h}^{j}\right\|_{L_{2}(\mathcal{D})},\left\|X_{h}^{j-1}\right\|_{L_{2}(\mathcal{D})}\right) P_{8 / 3}\left(\left\|X_{h}^{j}\right\|_{L_{4}(\mathcal{D})},\left\|X_{h}^{j-1}\right\|_{L_{4}(\mathcal{D})}\right) \\
& \quad+\left\langle Y_{h}^{j-1}, \Delta W^{j}\right\rangle+C\left\|X_{h}^{j}-X_{h}^{j-1}\right\|^{2}
\end{aligned}
$$

Summing with respect to $j$ then yields

$$
\begin{aligned}
J\left(X_{h}^{n}\right) & +\sum_{j=1}^{n}\left(\left|X_{h}^{j}-X_{h}^{j-1}\right|_{1}^{2}+k\left|Y_{h}^{j}\right|_{1}^{2}\right) \\
\leq & C\left(J\left(X_{h}^{0}\right)+\sum_{j=1}^{n}\left\|X_{h}^{j}-X_{h}^{j-1}\right\|^{2}\right. \\
& +\sum_{j=1}^{n}\left|\Delta W^{j}\right|_{1}^{2} P_{4 / 3}\left(\left\|X_{h}^{j}\right\|_{L_{2}(\mathcal{D})},\left\|X_{h}^{j-1}\right\|_{L_{2}(\mathcal{D})}\right) P_{8 / 3}\left(\left\|X_{h}^{j}\right\|_{L_{4}(\mathcal{D})},\left\|X_{h}^{j-1}\right\|_{L_{4}(\mathcal{D})}\right) \\
& \left.+\sum_{j=1}^{n}\left\langle Y_{h}^{j-1}, \Delta W^{j}\right\rangle\right) .
\end{aligned}
$$

It follows in a similar way as in the proof of Lemma 4.1. using also (4.12) with $\epsilon=1$ and $\delta>0$ so small that the third term on the right hand side above can be 
absorbed into the third term in the left hand side below, that

$$
\begin{aligned}
& \mathbf{E} \sup _{1 \leq n \leq N} J\left(X_{h}^{n}\right)^{p}+\mathbf{E}\left(\sum_{j=1}^{N} k\left|Y_{h}^{j}\right|_{1}^{2}\right)^{p} \leq C\left\{1+J\left(X_{h}^{0}\right)^{p}\right. \\
& +N^{p-1} \mathbf{E} \sum_{j=1}^{N}\left(\left|\Delta W^{j}\right|_{1}^{2 p} P_{4 p / 3}\left(\left\|X_{h}^{j}\right\|_{L_{2}(\mathcal{D})},\left\|X_{h}^{j-1}\right\|_{L_{2}(\mathcal{D})}\right)\right. \\
& \left.\left.\quad \times P_{8 p / 3}\left(\left\|X_{h}^{j}\right\|_{L_{4}(\mathcal{D})},\left\|X_{h}^{j-1}\right\|_{L_{4}(\mathcal{D})}\right)\right)+\mathbf{E} \sup _{1 \leq n \leq N}\left|\sum_{j=1}^{n}\left\langle Y_{h}^{j-1}, \Delta W^{j}\right\rangle\right|^{p}\right\} .
\end{aligned}
$$

The first three terms to the right of the inequality are bounded by assumption. For the fourth term we use that $\mathbf{E}\left|\Delta W^{j}\right|_{1}^{2 p} \leq C k^{p}\left\|A^{1 / 2} Q^{1 / 2}\right\|_{\mathrm{HS}}^{2 p}$, see (4.7), together with Hölder's inequality with conjugate exponents $q_{1}, q_{1}^{\prime}>1$, to get

$$
\begin{gathered}
N^{p-1} \mathbf{E} \sum_{j=1}^{N}\left(\left|\Delta W^{j}\right|_{1}^{2 p} P_{4 p / 3}\left(\left\|X_{h}^{j}\right\|_{L_{2}(\mathcal{D})},\left\|X_{h}^{j-1}\right\|_{L_{2}(\mathcal{D})}\right)\right. \\
\left.\times P_{8 p / 3}\left(\left\|X_{h}^{j}\right\|_{L_{4}(\mathcal{D})},\left\|X_{h}^{j-1}\right\|_{L_{4}(\mathcal{D})}\right)\right) \\
\leq N^{p-1} \sum_{j=1}^{N}\left(\mathbf{E}\left|\Delta W^{j}\right|_{1}^{2 p q_{1}^{\prime}}\right)^{1 / q_{1}^{\prime}}\left[\mathbf { E } \left(P_{4 p q_{1} / 3}\left(\left\|X_{h}^{j}\right\|_{L_{2}(\mathcal{D})},\left\|X_{h}^{j-1}\right\|_{L_{2}(\mathcal{D})}\right)\right.\right. \\
\left.\left.\times P_{8 p q_{1} / 3}\left(\left\|X_{h}^{j}\right\|_{L_{4}(\mathcal{D})},\left\|X_{h}^{j-1}\right\|_{L_{4}(\mathcal{D})}\right)\right)\right]^{1 / q_{1}} \\
\leq C\left\|A^{1 / 2} Q^{1 / 2}\right\|_{\mathrm{HS}}^{2 p} k^{p-1} N^{p-1} \sum_{j=1}^{N} k\left[\mathbf { E } \left(P_{4 p q_{1} / 3}\left(\left\|X_{h}^{j}\right\|_{L_{2}(\mathcal{D})},\left\|X_{h}^{j-1}\right\|_{L_{2}(\mathcal{D})}\right)\right.\right. \\
\left.\left.\times P_{8 p q_{1} / 3}\left(\left\|X_{h}^{j}\right\|_{L_{4}(\mathcal{D})},\left\|X_{h}^{j-1}\right\|_{L_{4}(\mathcal{D})}\right)\right)\right]^{1 / q_{1}}
\end{gathered}
$$

Here $\left\|A^{1 / 2} Q^{1 / 2}\right\|_{\mathrm{HS}}^{2 p} \leq C$. Hölder's inequality, now with $q_{2}, q_{2}^{\prime}>1$, bounds the above quantity by

$$
\begin{gathered}
\leq C T^{p-1} \sum_{j=1}^{N} k\left[\left(\mathbf{E}\left(P_{4 p q_{1} q_{2}^{\prime} / 3}\left(\left\|X_{h}^{j}\right\|_{L_{2}(\mathcal{D})},\left\|X_{h}^{j-1}\right\|_{L_{2}(\mathcal{D})}\right)\right)\right)^{1 / q_{2}^{\prime}}\right. \\
\left.\times\left(\mathbf{E}\left(P_{8 p q_{1} q_{2} / 3}\left(\left\|X_{h}^{j}\right\|_{L_{4}(\mathcal{D})},\left\|X_{h}^{j-1}\right\|_{L_{4}(\mathcal{D})}\right)\right)\right)^{1 / q_{2}}\right]^{1 / q_{1}} \\
\leq C\left\{1+\sum_{j=1}^{N} k\left(\mathbf{E}\left(P_{4 p q_{1} q_{2}^{\prime} / 3}\left(\left\|X_{h}^{j}\right\|_{L_{2}(\mathcal{D})},\left\|X_{h}^{j-1}\right\|_{L_{2}(\mathcal{D})}\right)\right)\right)^{1 / q_{2}^{\prime}}\right. \\
\left.\times\left(\mathbf{E}\left(P_{8 p q_{1} q_{2} / 3}\left(\left\|X_{h}^{j}\right\|_{L_{4}(\mathcal{D})},\left\|X_{h}^{j-1}\right\|_{L_{4}(\mathcal{D})}\right)\right)\right)^{1 / q_{2}}\right\} \\
\leq C\left\{1+\sum_{j=1}^{N} k\left[\mathbf{E}\left(P_{4 p q_{1} q_{2}^{\prime} / 3}\left(\left\|X_{h}^{j}\right\|_{L_{2}(\mathcal{D})},\left\|X_{h}^{j-1}\right\|_{L_{2}(\mathcal{D})}\right)\right)\right.\right. \\
\left.\left.+\mathbf{E}\left(P_{8 p q_{1} q_{2} / 3}\left(\left\|X_{h}^{j}\right\|_{L_{4}(\mathcal{D})},\left\|X_{h}^{j-1}\right\|_{L_{4}(\mathcal{D})}\right)\right)\right]\right\} .
\end{gathered}
$$


With $q_{1} q_{2}=3 / 2$ we use (1.2) to get

$$
\begin{aligned}
P_{8 p q_{1} q_{2} / 3}\left(\left\|X_{h}^{j}\right\|_{L_{4}(\mathcal{D})},\left\|X_{h}^{j-1}\right\|_{L_{4}(\mathcal{D})}\right) & \leq C\left(\left\|X_{h}^{j}\right\|_{L_{4}(\mathcal{D})}^{4 p}+\left\|X_{h}^{j-1}\right\|_{L_{4}(\mathcal{D})}^{4 p}+1\right) \\
& \leq C\left(\mathcal{F}\left(X_{h}^{j}\right)^{p}+\mathcal{F}\left(X_{h}^{j-1}\right)^{p}+1\right) .
\end{aligned}
$$

Furthermore,

$$
\begin{aligned}
& \sum_{j=1}^{N} k {\left[\mathbf { E } \left(P_{4 p q_{1} q_{2}^{\prime} / 3}\left(\left\|X_{h}^{j}\right\|_{L_{2}(\mathcal{D})},\left\|X_{h}^{j-1}\right\|_{L_{2}(\mathcal{D})}\right)\right.\right.} \\
& \leq C \mathbf{E} k \sum_{j=1}^{N}\left(\left\|X_{h}^{j}\right\|_{L_{2}(\mathcal{D})}^{4 p q_{1} q_{2}^{\prime} / 3}+\left\|X_{h}^{j-1}\right\|_{L_{2}(\mathcal{D})}^{4 p q_{1} q_{2}^{\prime} / 3}+1\right) \\
&=C T+C\left\|X_{h}^{0}\right\|_{L_{2}(\mathcal{D})}^{q}+C \mathbf{E} k \sum_{j=1}^{N}\left\|X_{h}^{j}\right\|_{L_{2}(\mathcal{D})}^{q},
\end{aligned}
$$

where for brevity $q=4 p q_{1} q_{2}^{\prime} / 3$. Let $t, s>1$ be conjugate exponents. Then, by Hölder's and Young's inequalities,

$$
\begin{gathered}
\mathbf{E} k \sum_{j=1}^{N}\left\|X_{h}^{j}\right\|_{L_{2}(\mathcal{D})}^{q} \leq \mathbf{E}\left[\sup _{1 \leq j \leq N}\left(\left\|X_{h}^{j}\right\|_{L_{2}(\mathcal{D})}^{q-2}\right) k \sum_{j=1}^{N}\left\|X_{h}^{j}\right\|_{L_{2}(\mathcal{D})}^{2}\right] \\
\leq \mathbf{E}\left[\sup _{1 \leq j \leq N}\left\|X_{h}^{j}\right\|_{L_{2}(\mathcal{D})}^{t(q-2)}\right]^{1 / t}\left[\mathbf{E}\left(k \sum_{j=1}^{N}\left\|X_{h}^{j}\right\|_{L_{2}(\mathcal{D})}^{2}\right)^{s}\right]^{1 / s} \\
\leq C\left(\mathbf{E}\left[\sup _{1 \leq j \leq N}\left\|X_{h}^{j}\right\|_{L_{2}(\mathcal{D})}^{t(q-2)}\right]+\mathbf{E}\left(k \sum_{j=1}^{N}\left\|X_{h}^{j}\right\|_{L_{2}(\mathcal{D})}^{2}\right)^{s}\right) \\
\quad \leq C\left(\mathbf{E}\left[\sup _{1 \leq j \leq N}\left\|X_{h}^{j}\right\|_{L_{2}(\mathcal{D})}^{t(q-2)}\right]+\mathbf{E}\left(k \sum_{j=1}^{N}\left|X_{h}^{j}\right|_{1}^{2}\right)^{s}\right)
\end{gathered}
$$

Next, (4.12) from Lemma 4.2 and (4.2) from Lemma 4.1 implies that

$$
\mathbf{E} k \sum_{j=1}^{N}\left\|X_{h}^{j}\right\|_{L_{2}(\mathcal{D})}^{q} \leq C+K \delta \mathbf{E}\left(\sum_{j=1}^{N} k\left|Y_{h}^{j}\right|_{1}^{2}\right)^{\frac{t(q-2)(1+\epsilon)}{4}} .
$$

We will find $\epsilon>0, q_{1}, q_{2}, q_{2}^{\prime}, t>1$, such that $q_{2}, q_{2}^{\prime}$ are conjugate, $q_{1} q_{2}=3 / 2$ and

$$
\frac{t\left(4 p q_{1} q_{2}^{\prime} / 3-2\right)(1+\epsilon)}{4} \leq p
$$

Since $q_{2}, q_{2}^{\prime}$ are conjugate and $q_{1} q_{2}=3 / 2$ we have that $q_{1} q_{2}^{\prime}=3 / 2\left(q_{2}^{\prime}-1\right)$ and hence (4.26) becomes

$$
t p\left(q_{2}^{\prime}-1\right) \frac{1+\epsilon}{2}-\frac{1+\epsilon}{2} \leq p
$$

Note that $q_{2}<3 / 2$ and hence $q_{2}^{\prime}>3$. If we set $q_{2}^{\prime}=3+\frac{1}{p^{2}}$ (and thus $q_{2}=\frac{3 p^{2}+1}{2 p^{2}+1}$ and $\left.q_{1}=\frac{6 p^{2}+3}{6 p^{2}+2}\right)$ we need to find $\epsilon>0$ and $t>1$ such that

$$
t p(1+\epsilon)+t \frac{1+\epsilon}{2 p}-\frac{1+\epsilon}{2} \leq p .
$$


But

$$
t p(1+\epsilon)+t \frac{1+\epsilon}{2 p}-\frac{1+\epsilon}{2} \rightarrow p+\frac{1}{2 p}-\frac{1}{2}<p
$$

as $\epsilon \rightarrow 0+$ and $t \rightarrow 1+$ and hence there is $\epsilon>0$ and $t>1$ such that (4.27) and hence (4.26) holds. Therefore, we can conclude from (4.24) and (4.25) that there is $\epsilon>0, q_{1}, q_{2}, q_{2}^{\prime}, t>1$, such that $q_{2}, q_{2}^{\prime}$ are conjugate, $q_{1} q_{2}=3 / 2$ and

$$
\begin{aligned}
& \sum_{j=1}^{N} k\left[\mathbf { E } \left(P_{4 p q_{1} q_{2}^{\prime} / 3}\left(\left\|X_{h}^{j}\right\|_{L_{2}(\mathcal{D})},\left\|X_{h}^{j-1}\right\|_{L_{2}(\mathcal{D})}\right) \leq C T+C\left\|X_{h}^{0}\right\|_{L_{2}(\mathcal{D})}^{4 p+\frac{2}{p^{2}}}\right.\right. \\
& \quad+K \delta\left(1+\mathbf{E}\left(\sum_{j=1}^{N} k\left|Y_{h}^{j}\right|_{1}^{2}\right)^{p}\right)
\end{aligned}
$$

Thus inserting (4.23) and (4.28) into (4.22) we get, with $C=C(T, p)>0$ and $K=K(T, p)>0$ independent of $\delta>0$,

$$
\begin{aligned}
& N^{p-1} \mathbf{E} \sum_{j=1}^{N}\left|\Delta W^{j}\right|_{1}^{2 p} P_{4 p / 3}\left(\left\|X_{h}^{j}\right\|_{L_{2}(\mathcal{D})}\right) P_{4 p / 3}\left(\left\|X_{h}^{j}\right\|_{L_{4}(\mathcal{D})}\right) \\
& \quad \leq C\left(1+\left\|X_{h}^{0}\right\|_{L_{2}(\mathcal{D})}^{4 p+\frac{2}{p^{2}}}+\sum_{j=0}^{N} k \mathbf{E} \mathcal{F}\left(X_{h}^{j}\right)^{p}\right)+K \delta\left(1+\mathbf{E}\left(\sum_{j=1}^{N} k\left|Y_{h}^{j}\right|_{1}^{2}\right)^{p}\right) .
\end{aligned}
$$

It remains to treat the Itô integral in (4.21). For this we invoke the Cauchy inequality and Burkholder-Davis-Gundy inequality to conclude that

$$
\begin{aligned}
& \mathbf{E} \sup _{1 \leq n \leq N}\left|\sum_{j=1}^{n}\left\langle Y_{h}^{j-1}, \Delta W^{j}\right\rangle\right|^{p} \\
& \leq C\left(1+\epsilon^{\prime} \mathbf{E} \sup _{0 \leq n \leq N}\left|\sum_{j=1}^{n}\left\langle Y_{h}^{j-1}, \Delta W^{j}\right\rangle\right|^{2 p}\right) \\
& \leq C\left(1+\epsilon^{\prime} \mathbf{E}\left(\sum_{j=1}^{N} k\left\|Q^{1 / 2} Y_{h}^{j-1}\right\|^{2}\right)^{p}\right) \\
& \leq C\left(1+\epsilon^{\prime} \mathbf{E}\left(\sum_{j=1}^{N} k\left\|Q^{1 / 2} A^{-1 / 2} P\right\|\left|Y_{h}^{j-1}\right|_{1}^{2}\right)^{p}\right) .
\end{aligned}
$$

Thus, since $\left\|Q^{1 / 2} A^{-1 / 2} P\right\|<\infty$, if we take $\epsilon^{\prime}>0$ small enough in (4.30) and $\delta>0$ small enough in (4.29), from (4.21) we may conclude that

$$
\begin{aligned}
\mathbf{E} \sup _{1 \leq n \leq N} J\left(X_{h}^{n}\right)^{p}+\mathbf{E}\left(\sum_{j=1}^{N} k\left|Y_{h}^{j}\right|_{1}^{2}\right)^{p} \\
\leq C\left(1+J\left(X_{h}^{0}\right)^{p}+\left\|X_{h}^{0}\right\|_{L_{2}(\mathcal{D})}^{4 p+\frac{1}{p^{2}}}+k \mathcal{F}\left(X_{h}^{0}\right)^{p}+k\left|Y_{h}^{0}\right|_{1}^{2 p}\right. \\
\left.\quad+\sum_{j=1}^{N} k \mathbf{E} \sup _{1 \leq n \leq j} \mathcal{F}\left(X_{h}^{n}\right)^{p}\right) .
\end{aligned}
$$


Thus, if $C k<1$, then the desired result follows from Gronwall's lemma by noting that $\mathcal{F}(u) \leq J(u)$.

\section{Convergence}

Recall from Theorem 3.1 that $X$ satisfies the mild equation

$$
X(t)=E(t) X_{0}-\int_{0}^{t} A E(t-s) f(X(s)) \mathrm{d} s+\int_{0}^{t} E(t-s) \mathrm{d} W(s) .
$$

Similarly, equation (1.6) has the mild formulation

$$
X_{h}^{n}=R_{k, h}^{n} P_{h} X_{0}-\sum_{j=1}^{n} A_{h} R_{k, h}^{n-j+1} P_{h} f\left(X_{h}^{j}\right)+\sum_{j=1}^{n} R_{k, h}^{n-j+1} P_{h} \Delta W^{j}
$$

where $R_{k, h}^{n}=\left(I+k A_{h}^{2}\right)^{-n}$.

Remark 5.1. Preservation of mass. From (2.5), (5.1) and (2.9), (5.2) it follows that if $W(t)$ has zero average, i.e., $(I-P) W(t)=0$, then $(I-P) X(t)=(I-P) X_{0}$ and $(I-P) X_{h}^{n}=(I-P) X_{0}$. This means that $X(t)$ and $X_{h}^{n}$ preserve the mass.

In order to prove convergence of $X_{h}^{n}$, we first state a maximal type error estimate for the stochastic convolution. We define the backward Euler approximation of the stochastic convolution $W_{A}(t):=\int_{0}^{t} E(t-s) \mathrm{d} W(s)$ by

$$
W_{A_{h}}^{n}:=\sum_{j=1}^{n} R_{k, h}^{n-j+1} P_{h} \Delta W^{j}=\sum_{j=1}^{n} \int_{t_{j-1}}^{t_{j}} R_{k, h}^{n-j+1} P_{h} \mathrm{~d} W(s) .
$$

Lemma 5.2. Let $\gamma \in\left(0, \frac{1}{2}\right], \beta \in[1,2]$, and $p \geq 1$. Then there is $C=C(p, \gamma, T)$ such that for $h, k>0$

$$
\left(\mathbf{E}\left(\sup _{0 \leq n \leq N}\left\|W_{A}\left(t_{n}\right)-W_{A_{h}}^{n}\right\|^{p}\right)\right)^{1 / p} \leq C\left(h^{\beta}+k^{\beta / 4}\right)\left\|A^{(\beta-2) / 2+\gamma} P Q^{1 / 2}\right\|_{\mathrm{HS}}
$$

Proof. The proof is completely analogous to the proofs of [20, Proposition 5.1] and [23. Theorem 2.1], based on a discrete factorization method using the analyticity of the semigroup $E$ and the deterministic error estimate

$$
\left\|\left(E\left(t_{n}\right)-R_{k, h}^{n}\right) P_{h} v\right\| \leq C\left(h^{\beta}+k^{\beta / 4}\right) t_{n}^{-(\beta-\alpha) / 4}|v|_{\alpha}, \quad t_{n}>0
$$

for $\beta \in[1,2], \alpha \in[-1,1]$, from [12, Lemma 5.5] and [25, Theorem 2.2].

Remark 5.3. In [20, Proposition 5.1], instead of the term $\left\|A^{(\beta-2) / 2+\gamma} P Q^{1 / 2}\right\|_{\mathrm{HS}}$ which appears on the right hand side of (5.3), one has $\left\|A^{(\beta-1) / 2+\gamma} Q^{1 / 2}\right\|_{\mathrm{HS}}$. The reason for this difference (besides the different boundary conditions) is that [20] considers the stochastic Allen-Cahn equation where the semigroup in the stochastic convolution is generated by the Laplacian $\Delta$ which has a weaker smoothing effect than in the present case, where the semigroup is generated by $-\Delta^{2}$. The proofs in [20] and [23] require that $p$ is large, but the result is then valid for smaller $p \geq 1$ as well. 
Lemma 5.4. Let $0<\delta<1$. The following deterministic error estimates hold for $v \in H$ :

$$
\begin{gathered}
\left\|A_{h} R_{k, h}^{n} P_{h} v-A_{h} E_{h}\left(t_{n}\right) P_{h} v\right\| \leq C k^{\frac{1}{2}(1-\delta)} t_{n}^{-1+\frac{\delta}{2}}\|v\|, \quad t_{n}>0 \text { and } h, k>0, \\
\left\|A_{h} E_{h}(t) P_{h} v-A E(t) v\right\| \leq C h^{2(1-\delta)} t^{-1+\frac{\delta}{2}}\|v\|, \quad t>0 \text { and } h>0 .
\end{gathered}
$$

Proof. Note that it is enough to consider $v \in \dot{H}$, as for $v$ constant the above differences equal 0 . The error bounds follow by a simple interpolation between the cases $\delta=0$ and $\delta=1$. For $\delta=1$ we use the estimates from (2.6) and (2.10) to get

$$
\left\|A_{h} R_{k, h}^{n} P_{h}\right\|+\left\|A_{h} E_{h}\left(t_{n}\right) P_{h}\right\| \leq C t_{n}^{-1 / 2}, \quad\left\|A_{h} E_{h}(t) P_{h}\right\|+\|A E(t)\| \leq C t^{-1 / 2} .
$$

Estimate (5.5) with $\delta=0$ follows by expansion in the eigenbasis of $A_{h}$ and Parseval's identity. For estimate (5.6) with $\delta=0$, we first write, for $v \in \dot{H}$,

$$
\begin{aligned}
\left\|A_{h} E_{h}(t) P_{h} v-A E(t) v\right\| \leq\left\|\left(A_{h}^{2} E_{h}(t) P_{h}-A^{2} E(t)\right) A^{-1} v\right\| \\
\quad+\left\|A_{h}^{2} E_{h}(t) P_{h}\left(A_{h}^{-1} P_{h}-A^{-1}\right) v\right\| \\
=\left\|D_{t}\left(E_{h}(t) P_{h}-E(t)\right) A^{-1} v\right\|+\left\|A_{h}^{2} E_{h}(t) P_{h}\left(R_{h}-I\right) A^{-1} v\right\| .
\end{aligned}
$$

where we used the identity $R_{h}=A_{h}^{-1} P_{h} A$. The desired bound $C t^{-1} h^{2}\left\|A\left(A^{-1} v\right)\right\|=$ $C h^{2} t^{-1}\|v\|$ for the last term follows immediately from (2.12) and (2.10). The first term is an error estimate for the time derivative of the solution of the linear CahnHilliard equation with smooth initial-value $u_{0}=A^{-1} v \in \dot{H}^{2}$. To prove this we adapt the arguments in [27, Chapt. 3] and [12, Sect. 5], where error estimates with lower initial regularity are proved. Let $u(t)=E(t) u_{0}, u_{h}(t)=E_{h}(t) P_{h} u_{0}$. Then the error $e=u_{h}-u$ satisfies the equation, see [12, (5.4)],

$$
G_{h}^{2} \dot{e}+e=\rho+G_{h} \eta, t>0 ; \quad P_{h} e(0)=0,
$$

with

$$
G_{h}=A_{h}^{-1} P_{h}, \quad R_{h}=G_{h} A, \quad \rho=\left(R_{h}-I\right) u, \quad \eta=-\left(R_{h}-I\right) A^{-1} \dot{u} .
$$

Any solution of an equation of the form (5.7) satisfies the following bound, with arbitrary $\epsilon>0$,

$$
\|e(t)\| \leq \epsilon \sup _{s \in[0, t]} s\|\dot{\rho}(s)\|+C_{\epsilon} \sup _{s \in[0, t]}\|\rho(s)\|+\left(\int_{0}^{t}\|\eta(s)\|^{2} \mathrm{~d} s\right)^{1 / 2} .
$$

In order to prove this we let $e_{1}$ be the solution of (5.7) with only $\rho$ as the source term and $P_{h} e_{1}(0)=0$. Moreover, we let $e_{2}$ solve the same equation but driven by $G_{h} \eta$ alone and with $e_{2}(0)=0$. Then $e=e_{1}+e_{2}$ solves (5.7). We quote a bound for $e_{1}$ from [27, Lemma 3.5]:

$$
\left\|e_{1}(t)\right\| \leq \epsilon \sup _{s \in[0, t]} s\|\dot{\rho}(s)\|+C_{\epsilon} \sup _{s \in[0, t]}\|\rho(s)\| .
$$

In order to quote this lemma we note that $G_{h}$ is selfadjoint, positive semidefinite on $\dot{H}$ and that $G_{h} e_{1}(0)=A_{h}^{-1} P_{h} e_{1}(0)=0$. For $e_{2}$ we have

$$
\left\|e_{2}(t)\right\| \leq\left(\int_{0}^{t}\|\eta(s)\|^{2} \mathrm{~d} s\right)^{1 / 2} .
$$

This is proved by a simple energy argument, see the beginning of the proof of [12, Lemma 5.2]. The reason why we need different proofs for $e_{1}$ and $e_{2}$ is that $G_{h}$ in front of $\eta$ must not appear in (5.9) for we have good bounds for $\eta$ but not for $G_{h} \eta$. 
This proves (5.9), which can now be combined with bounds for $\rho$ and $\eta$, obtained from bounds for $R_{h}$ and regularity estimates for $u=E(t) u_{0}$, to get an error bound for $\|e(t)\|$. However, we aim for $\|\dot{e}(t)\|$ and therefore take the derivative of the equation in (5.7) and multiply by $t$ to obtain an equation for $t \dot{e}(t)$ :

$$
t G_{h}^{2} \ddot{e}+t \dot{e}=t \dot{\rho}+t G_{h} \dot{\eta}
$$

which can be written as

$$
G_{h}^{2}(t \dot{e})^{\cdot}+(t \dot{e})=G_{h}^{2} \dot{e}+t \dot{\rho}+G_{h}(t \dot{\eta})=-e+\rho+t \dot{\rho}+G_{h}(\eta+t \dot{\eta}),
$$

where we substituted $G_{h}^{2} \dot{e}=-e+\rho+G_{h} \eta$ from (5.7). Thus, $t \dot{e}$ satisfies an equation of the form (5.7) but with $\rho$ and $\eta$ replaced by $-e+\rho+t \dot{\rho}$ and $\eta+t \dot{\eta}$. An application of (5.9) with $\epsilon=\frac{1}{2}$, say, gives

$$
\begin{aligned}
t\|\dot{e}(t)\| \leq & \frac{1}{2} \sup _{s \in[0, t]}\left(s\|\dot{e}(s)\|+2 s\|\dot{\rho}(s)\|+s^{2}\|\ddot{\rho}(s)\|\right) \\
& +C \sup _{s \in[0, t]}(\|e(s)\|+\|\rho(s)\|+s\|\dot{\rho}(s)\|) \\
& +\left(\int_{0}^{t}\left(\|\eta(s)\|^{2}+s^{2}\|\dot{\eta}(s)\|^{2}\right) \mathrm{d} s\right)^{1 / 2} .
\end{aligned}
$$

Since $t$ is arbitrary here we may apply a standard kick-back argument to remove the term $s\|\dot{e}(s)\|$. Another application of (5.9), now with $\epsilon=1$, takes care of the term $\|e(s)\|$, which leads to

$$
\begin{aligned}
t\|\dot{e}(t)\| \leq & C \sup _{s \in[0, t]}\left(\|\rho(s)\|+s\|\dot{\rho}(s)\|+s^{2}\|\ddot{\rho}(s)\|\right) \\
& +C\left(\int_{0}^{t}\left(\|\eta(s)\|^{2}+s^{2}\|\dot{\eta}(s)\|^{2}\right) \mathrm{d} s\right)^{1 / 2} .
\end{aligned}
$$

Here we use (2.12) and recall the regularity estimates (2.6), (2.7) for $u(t)=$ $E(t) A^{-1} v$ :

$$
s^{j}\left\|D_{s}^{j} \rho(s)\right\|=s^{j}\left\|\left(R_{h}-I\right) D_{s}^{j} u(s)\right\| \leq C h^{2} s^{j}\left\|A D_{s}^{j} E(s) A^{-1} v\right\| \leq C h^{2}\|v\|
$$

and

$$
\begin{aligned}
& \left(\int_{0}^{t} s^{2 j}\left\|D_{s}^{j} \eta(s)\right\|^{2} \mathrm{~d} s\right)^{1 / 2}=\left(\int_{0}^{t} s^{2 j}\left\|\left(R_{h}-I\right) A^{-1} D_{s}^{j} \dot{u}(s)\right\|^{2} \mathrm{~d} s\right)^{1 / 2} \\
& \quad \leq C h^{2}\left(\int_{0}^{t} s^{2 j}\left\|D_{s}^{j+1} u(s)\right\|^{2} \mathrm{~d} s\right)^{1 / 2} \\
& \quad \leq C h^{2}\left(\int_{0}^{t} s^{2 j}\left\|A^{2 j} E(s) A^{-1} v\right\|^{2} \mathrm{~d} s\right)^{1 / 2} \\
& \quad \leq C h^{2}\left(\int_{0}^{t} s^{2 j}\left\|A^{2 j+1} E(s) v\right\|^{2} \mathrm{~d} s\right)^{1 / 2} \leq C h^{2}\|v\| .
\end{aligned}
$$

This completes the proof.

Theorem 5.5. Suppose that (4.14) holds, $\left\|A^{1 / 2} Q^{1 / 2}\right\|_{\mathrm{HS}}<\infty, \beta \in[1,2]$, and that $\left|X_{0}\right|_{\beta}<\infty$. Let $h>0$ and $k>0$ be small and $0<\epsilon, \delta<1$. Then, there is $\Omega_{h, k}^{\epsilon} \subset \Omega$ with $\mathbf{P}\left(\Omega_{h, k}^{\epsilon}\right)>1-\epsilon$, and $C=C(T, \epsilon, \delta)$ such that for all $\omega \in \Omega_{h, k}^{\epsilon}$,

$$
\left\|X\left(t_{n}\right)-X_{h}^{n}\right\| \leq C\left(\left(h^{\beta}+k^{\beta / 4}\right)\left|X_{0}\right|_{\beta}+h^{2(1-\delta)}+k^{\frac{1}{2}(1-\delta)}\right), \quad t_{n} \in[0, T] .
$$


Proof. It follows from Proposition 3.2 for (5.10), Theorem 3.1 for (5.11), Theorem 4.3 for (5.12), and Lemma 5.2 for (5.13) that for every $0<\epsilon, \delta<1$ and $h, k>0$ small enough, there is $\Omega_{h, k}^{\epsilon} \subset \Omega$ with $\mathbf{P}\left(\Omega_{h, k}^{\epsilon}\right)>1-\epsilon$ and $K_{T, \epsilon}>0$ such that

$$
\begin{array}{rlrl}
\|X(t)-X(s)\| & \leq K_{T, \epsilon}|t-s|^{\frac{1}{2}(1-\delta)}, & & t, s \in[0, T], \omega \in \Omega_{h, k}^{\epsilon}, \\
|X(t)|_{1}^{2}+\|X(t)\|_{L_{4}}^{4} & \leq K_{T, \epsilon}, & & t \in[0, T], \omega \in \Omega_{h, k}^{\epsilon}, \\
\left|X_{h}^{n}\right|_{1}^{2}+\left\|X_{h}^{n}\right\|_{L_{4}}^{4} \leq K_{T, \epsilon}, & & t_{n} \in[0, T], \omega \in \Omega_{h, k}^{\epsilon} \\
\left\|W_{A}\left(t_{n}\right)-W_{A_{h}}^{n}\right\| & \leq K_{T, \epsilon}\left(h^{2}+k^{1 / 2}\right), & & t_{n} \in[0, T], \omega \in \Omega_{h, k}^{\epsilon}, .
\end{array}
$$

Note that it is enough to establish the above four bounds individually with $\epsilon / 4$ on $\Omega_{h, k}^{\epsilon / 4, i}, i=1, \ldots, 4$, and then set $\Omega_{h, k}^{\epsilon}=\cap_{i=1}^{4} \Omega_{h, k}^{\epsilon / 4, i}$. The estimate in (5.10) follows directly from the assumption on the initial data and Proposition 3.2 The remaining bounds can be proved by using Chebychev's inequality together with bounds from Theorem 3.1, Theorem 4.3, and Lemma 5.2. For example, to prove (5.13), consider

$$
F_{h, k}:=\frac{\sup _{0 \leq n \leq N}\left\|W_{A}\left(t_{n}\right)-W_{A_{h}}^{n}\right\|}{h^{2}+k^{1 / 2}} .
$$

Chebychev's inequality and Lemma 5.2 for some $p \geq 1, \gamma=\frac{1}{2}$, and $\beta=2$, give

$$
\mathbf{P}\left(\left\{\omega \in \Omega: F_{h, k}>\alpha\right\}\right) \leq \frac{1}{\alpha^{p}} \mathbf{E}\left[F_{h, k}^{p}\right] \leq \frac{K^{p}}{\alpha^{p}}, \quad K=C\left\|A^{1 / 2} Q^{1 / 2}\right\|_{\mathrm{HS}}
$$

We choose $\alpha=\epsilon^{-1 / p} K$ and set $\Omega_{h, k}^{\epsilon}=\left\{\omega \in \Omega: F_{h, k} \leq \epsilon^{-1 / p} K\right\}$. Then

$$
\mathbf{P}\left(\Omega_{h, k}^{\epsilon}\right)=1-\mathbf{P}\left(\left\{\omega \in \Omega: F_{h, k}>\epsilon^{-1 / p} K\right\}\right) \geq 1-\epsilon,
$$

and (5.13) follows.

Now let $\omega \in \Omega_{h, k}^{\epsilon}$. We decompose the error $e_{n}:=X\left(t_{n}\right)-X_{h}^{n}$ as

$$
\begin{aligned}
e_{n}= & \left(E\left(t_{n}\right)-R_{k, h}^{n} P_{h}\right) X_{0} \\
& +\sum_{j=1}^{n} \int_{t_{j-1}}^{t_{j}}\left(A_{h} R_{k, h}^{n-j+1} P_{h} f\left(X_{h}^{j}\right)-A E\left(t_{n}-s\right) f(X(s))\right) \mathrm{d} s \\
& +W_{A}\left(t_{n}\right)-W_{A_{h}}^{n}=: e_{n}^{1}+e_{n}^{2}+e_{n}^{3} .
\end{aligned}
$$

For the first error term we use (5.4) to get

$$
\left\|e_{n}^{1}\right\| \leq C\left(h^{\beta}+k^{\beta / 4}\right)\left|X_{0}\right|_{\beta}
$$


The term $e_{n}^{2}$ is most involved and we decompose it further as

$$
\begin{aligned}
A_{h} R_{k, h}^{n-j+1} P_{h} f\left(X_{h}^{j}\right)-A E_{h}\left(t_{n}-s\right) f(X(s)) \\
=\left(A_{h} R_{k, h}^{n-j+1}-A_{h} E_{h}\left(t_{n}-t_{j-1}\right)\right) P_{h} f\left(X_{h}^{j}\right) \\
\quad+\left(A_{h} E_{h}\left(t_{n}-t_{j-1}\right) P_{h}-A E\left(t_{n}-t_{j-1}\right)\right) f\left(X_{h}^{j}\right) \\
\quad+A^{3 / 2} E\left(t_{n}-t_{j-1}\right) A^{-1 / 2} P\left(f\left(X_{h}^{j}\right)-f\left(X\left(t_{j}\right)\right)\right) \\
\quad+A\left(E\left(t_{n}-t_{j-1}\right)-E\left(t_{n}-s\right)\right) f\left(X\left(t_{j}\right)\right) \\
\quad+A^{3 / 2} E\left(t_{n}-s\right) A^{-1 / 2} P\left(f\left(X\left(t_{j}\right)\right)-f(X(s))\right) \\
=: e_{n, j}^{2,1}+e_{n, j}^{2,2}+e_{n, j}^{2,3}+e_{n, j}^{2,4}+e_{n, j}^{2,5} .
\end{aligned}
$$

Here we used (2.5) to obtain $A E(t)=A^{3 / 2} E(t) A^{-1 / 2} P$. Further, since $f$ is cubic we have $\|f(x)\| \leq C\left(1+|x|_{1}^{3}\right)$ by Hölder's and Sobolev's inequalities. Then by (5.5), and (5.12) it follows that, with $C=C(T, \epsilon, \delta)$,

$$
\begin{aligned}
\left\|e_{n, j}^{2,1}\right\| & \leq C k^{\frac{1}{2}(1-\delta)} t_{n-j+1}^{-1+\frac{\delta}{2}}\left\|f\left(X_{h}^{j}\right)\right\| \leq C k^{\frac{1}{2}(1-\delta)} t_{n-j+1}^{-1+\frac{\delta}{2}}\left(1+\left|X_{h}^{j}\right|_{1}^{3}\right) \\
& \leq C k^{\frac{1}{2}(1-\delta)} t_{n-j+1}^{-1+\frac{\delta}{2}} .
\end{aligned}
$$

Similarly, by (5.6) and (5.12),

$$
\left\|e_{n, j}^{2,2}\right\| \leq C h^{2(1-\delta)} t_{n-j+1}^{-1+\frac{\delta}{2}} .
$$

Also, using the local Lipschitz bound $|P(f(x)-f(y))|_{-1} \leq C\left(1+|x|_{1}^{2}+|y|_{1}^{2}\right)\|x-y\|$, cf. (4.18), together with (5.11), and (5.12), we obtain

$$
\begin{aligned}
\left\|e_{n, j}^{2,3}\right\| & \leq C\left(t_{n}-t_{j-1}\right)^{-3 / 4} \mid P\left(f\left(X_{h}^{j}\right)-\left.f\left(X\left(t_{j}\right)\right)\right|_{-1}\right. \\
& \leq C t_{n-j+1}^{-3 / 4}\left(1+\left|X_{h}^{j}\right|_{1}^{2}+\left|X\left(t_{j}\right)\right|_{1}^{2}\right)\left\|X_{h}^{j}-X\left(t_{j}\right)\right\| \\
& \leq C t_{n-j+1}^{-3 / 4}\left\|e_{j}\right\| .
\end{aligned}
$$

Furthermore, for $s \in\left[t_{j-1}, t_{j}\right]$, by (2.6) and $\|(E(t)-I) x\| \leq C t^{\frac{1}{2}(1-\delta)}\left\|A^{1-\delta} x\right\|$, we have

$$
\begin{aligned}
\left\|e_{n, j}^{2,4}\right\| & =\left\|\left(E\left(s-t_{j-1}\right)-I\right) A^{-1+\delta} A^{2-\delta} E\left(t_{n}-s\right) f\left(X\left(t_{j}\right)\right)\right\| \\
& \leq C\left(s-t_{j-1}\right)^{\frac{1}{2}(1-\delta)}\left(t_{n}-s\right)^{-1+\frac{\delta}{2}}\left\|f\left(X\left(t_{j}\right)\right)\right\| \\
& \leq C k^{\frac{1}{2}(1-\delta)}\left(t_{n}-s\right)^{-1+\frac{\delta}{2}} .
\end{aligned}
$$

Using also (5.10), for $s \in\left[t_{j-1}, t_{j}\right]$, we have

$$
\begin{aligned}
\left\|e_{n, j}^{2,5}\right\| & \leq C\left(t_{n}-s\right)^{-3 / 4}\left(1+\left|X\left(t_{j}\right)\right|_{1}^{2}+|X(s)|_{1}^{2}\right)\left\|X\left(t_{j}\right)-X(s)\right\| \\
& \leq C\left(t_{n}-s\right)^{-3 / 4}\left(t_{j}-s\right)^{\frac{1}{2}(1-\delta)} \leq C\left(t_{n}-s\right)^{-3 / 4} k^{\frac{1}{2}(1-\delta)} .
\end{aligned}
$$

Finally, by (5.13), $\left\|e_{n}^{3}\right\| \leq C\left(h^{2}+k^{1 / 2}\right)$. Collecting all the above terms and applying a generalized version of Gronwall's lemma, [12, Lemma 7.1], finishes the proof if $k$ is small enough.

Theorem 5.6. Under the hypothesis of Theorem 5.5 with $\beta=1$, we have

$$
\lim _{h, k \rightarrow 0} \mathbf{E} \sup _{0 \leq n \leq N}\left\|X\left(t_{n}\right)-X_{h}^{n}\right\|^{2}=0 .
$$


Proof. It follows from Theorem 3.1 and Theorem 4.3 that there is $K>0$ such that

$$
\mathbf{E} \sup _{0 \leq n \leq N}\left(\left\|X\left(t_{n}\right)\right\|_{L_{4}}^{4}+\left\|X_{h}^{n}\right\|_{L_{4}}^{4}\right) \leq K .
$$

Let $\epsilon>0,0<h, k<1$ small enough, and let $C_{\epsilon}=C(T, \epsilon, \delta)$ and $\Omega_{h, k}^{\epsilon}$ as in Theorem 5.5. Then, by using Theorem 5.5 with $\beta=1$ and $\delta=\frac{1}{2}$, we get

$$
\begin{aligned}
\mathbf{E} \sup _{0 \leq n \leq N} & \left\|X\left(t_{n}\right)-X_{h}^{n}\right\|^{2} \leq \int_{\Omega_{h, k}^{\epsilon}} \sup _{0 \leq n \leq N}\left\|X\left(t_{n}\right)-X_{h}^{n}\right\|^{2} \mathrm{~d} \mathbf{P} \\
& +2 \int_{\left(\Omega_{h, k}^{\epsilon}\right)^{c}} \sup _{0 \leq n \leq N}\left(\left\|X\left(t_{n}\right)\right\|^{2}+\left\|X_{h}^{n}\right\|^{2}\right) \mathrm{d} \mathbf{P} \\
\leq & C_{\epsilon}\left(h^{2}+k^{1 / 2}\right)+4 \epsilon^{1 / 2}\left(\int_{\left(\Omega_{h, k}^{\epsilon}\right)^{c}} \sup _{0 \leq n \leq N}\left(\left\|X\left(t_{n}\right)\right\|^{4}+\left\|X_{h}^{n}\right\|^{4}\right) \mathrm{d} \mathbf{P}\right)^{1 / 2} \\
\leq & C_{\epsilon}\left(h^{2}+k^{1 / 2}\right)+4 \epsilon^{1 / 2}\left(\mathbf{E} \sup _{0 \leq n \leq N}\left(\left\|X\left(t_{n}\right)\right\|^{4}+\left\|X_{h}^{n}\right\|^{4}\right)\right)^{1 / 2} \\
\leq & C_{\epsilon}\left(h^{2}+k^{1 / 2}\right)+4 \epsilon^{1 / 2}|\mathcal{D}|^{1 / 2}\left(\mathbf{E} \sup _{0 \leq n \leq N}\left(\left\|X\left(t_{n}\right)\right\|_{L_{4}}^{4}+\left\|X_{h}^{n}\right\|_{L_{4}}^{4}\right)\right)^{1 / 2} \\
\leq & C_{\epsilon}\left(h^{2}+k^{1 / 2}\right)+4 \epsilon^{1 / 2}|\mathcal{D}|^{1 / 2} K .
\end{aligned}
$$

Let $\eta>0$. Choose $0<\epsilon<1$ such that $8 \epsilon^{1 / 2}|\mathcal{D}|^{1 / 2} K<\frac{\eta}{2}$. Therefore, if $\max (h, k)<$ $\left(\frac{\eta}{4 C_{\epsilon}}\right)^{2}$, then

$$
\mathbf{E} \sup _{0 \leq n \leq N}\left\|X\left(t_{n}\right)-X_{h}^{n}\right\|^{2}<\eta
$$

and the proof is complete.

Acknowledgement. We thank the anonymous referees for constructive criticism that helped to clarify the presentation of the paper.

\section{REFERENCES}

[1] S. Becker And A. Jentzen, Strong convergence rates for nonlinearity-truncated Eulertype approximations of stochastic Ginzburg-Landau equations. arXiv:1601.05756 2016, https://arxiv.org/abs/1601.05756

[2] D. BlÖmker And A. JentZen, Galerkin approximations for the stochastic Burgers equation, SIAM J. Numer. Anal., 51 (2013), pp. 694-715, https://doi.org/10.1137/110845756

[3] D. Blömker, M. Kamrani, and S. M. Hosseini, Full discretization of the stochastic Burgers equation with correlated noise, IMA J. Numer. Anal., 33 (2013), pp. 825-848, https://doi.org/10.1093/imanum/drs035.

[4] D. Blömker, S. Maier-PaApe, and T. Wanner, Spinodal decomposition for the stochastic Cahn-Hilliard equation, in International Conference on Differential Equations, Vol. 1, 2 (Berlin, 1999), World Sci. Publ., River Edge, NJ, 2000, pp. 1265-1267.

[5] Z. Brzeźniak, E. Carelli, and A. Prohl, Finite-element-based discretizations of the incompressible Navier-Stokes equations with multiplicative random forcing, IMA J. Numer. Anal., 33 (2013), pp. 771-824, https://doi.org/10.1093/imanum/drs032

[6] C. CARdon-Weber, Implicit approximation scheme for the Cahn-Hilliard stochastic equation. Preprint, Laboratoire des Probabilités et Modelèles Aléatoires, Université Paris V, 2000.

[7] E. Carelli and A. Prohl, Rates of convergence for discretizations of the stochastic incompressible Navier-Stokes equations, SIAM J. Numer. Anal., 50 (2012), pp. 2467-2496, https://doi.org/10.1137/110845008

[8] H. E. Cook, Brownian motion in spinodal decomposition, Acta Metallurgica, 18 (1970). 
[9] G. Da Prato and A. Debussche, Stochastic Cahn-Hilliard equation, Nonlinear Anal., 26 (1996), pp. 241-263, https://doi.org/10.1016/0362-546X(94)00277-0

[10] G. Da Prato and J. ZabczyK, Stochastic Equations in Infinite Dimensions, vol. 44 of Encyclopedia of Mathematics and its Applications, Cambridge University Press, Cambridge, 1992.

[11] E. B. Davies, Spectral Theory and Differential Operators, Cambridge Studies in Advanced Mathematics, 42, Cambridge University Press, Cambridge, 1995.

[12] C. M. Elliott and S. LaRsson, Error estimates with smooth and nonsmooth data for a finite element method for the Cahn-Hilliard equation, Math. Comp., 58 (1992), pp. 603-630, S33-S36, https://doi.org/10.2307/2153205.

[13] G. B. Folland, Real Analysis, John Wiley \& Sons, Inc., New York, 1999.

[14] I. GyÖNGY AND A. Millet, On discretization schemes for stochastic evolution equations, Potential Anal., 23 (2005), pp. 99-134, https://doi.org/10.1007/s11118-004-5393-6

[15] I. GyÖngy, S. Sabanis, And D. ŠIšKa, Convergence of tamed Euler schemes for a class of stochastic evolution equations, Stoch. Partial Differ. Equ. Anal. Comput., 4 (2016), pp. 225245 , https://doi.org/10.1007/s40072-015-0057-7

[16] M. HutzenthaleR AND A. Jentzen, On a perturbation theory and on strong convergence rates for stochastic ordinary and partial differential equations with non-globally monotone coefficients. arXiv:1401.0295, 2014, https://arxiv.org/abs/1401.0295

[17] M. Hutzenthaler, A. Jentzen, And D. SAlimova, Strong convergence of full-discrete nonlinearity-truncated accelerated exponential Euler-type approximations for stochastic Kuramoto-Sivashinsky equations. arXiv:1604.02053, 2016, https://arxiv.org/abs/1604.02053

[18] A. JENTZEN AND P. PUŠNIK, Strong convergence rates for an explicit numerical approximation method for stochastic evolution equations with non-globally Lipschitz continuous nonlinearities. arXiv:1504.03523, 2015, https://arxiv.org/abs/1504.03523

[19] G. T. KOSSIORIS AND G. E. ZOURARIS, Finite element approximations for a linear fourth-order parabolic SPDE in two and three space dimensions with additive space-time white noise, Appl. Numer. Math., 67 (2013), pp. 243-261, https://doi.org/10.1016/j.apnum.2012.01.003

[20] M. Kovács, S. LARSSOn, AND F. Lindgren, On the backward Euler approximation of the stochastic Allen-Cahn equation., J. Appl. Probab., 52 (2015), pp. 323-338, https://doi.org/10.1239/jap/1437658601

[21] M. Kovács, S. LARsson, AND F. LindGRen, On the discretisation in time of the stochastic Allen-Cahn equation. arXiv:1510.03684, 2015, https://arxiv.org/abs/1510.03684 To appear in Matematische Nachrichten.

[22] M. Kovács, S. LARsson, AND A. Mesforush, Finite element approximation of the Cahn-Hilliard-Cook equation, SIAM J. Numer. Anal., 49 (2011), pp. 2407-2429, https://doi.org/10.1137/110828150

[23] M. Kovács, S. Larsson, AND A. Mesforush, Erratum: Finite element approximation of the Cahn-Hilliard-Cook equation, SIAM J. Numer. Anal., 52 (2014), pp. 2594-2597, https://doi.org/10.1137/140968161

[24] R. KuRniaWAN, Numerical approximations of stochastic partial differential equations with non-globally Lipschitz continuous nonlinearities. Master thesis, ETH Zürich, 2014.

[25] S. LARSSON AND A. Mesforush, Finite-element approximation of the linearized Cahn-Hilliard-Cook equation, IMA J. Numer. Anal., 31 (2011), pp. 1315-1333, https://doi.org/10.1093/imanum/drq042

[26] J. PRINTEMS, On the discretization in time of parabolic stochastic partial differential equations, M2AN Math. Model. Numer. Anal., 35 (2001), pp. 1055-1078, https://doi.org/10.1051/m2an:2001148

[27] V. Thomée, Galerkin Finite Element Methods for Parabolic Problems, Springer-Verlag, Berlin, 2006. 
FINITE ELEMENT APPROXIMATION OF THE CAHN-HILLIARD-COOK EQUATION

Cybermedia Center, Osaka University, 1-32 Machikaneyama, Toyonaka, Osaka 5600043, JAPAN

E-mail address: furihata@cmc.osaka-u.ac.jp

Department of Mathematical Sciences, Chalmers University of Technology and University of Gothenburg, SE-412 96 Gothenburg, Sweden

E-mail address: mihaly@chalmers.se

Department of Mathematical Sciences, Chalmers University of Technology and University of Gothenburg, SE-412 96 Gothenburg, Sweden

E-mail address: stig@chalmers.se

Cybermedia Center, Osaka University, 1-32 Machikaneyama, Toyonaka, Osaka 5600043, JAPAN

E-mail address: fredrik.lindgren1979@gmail.com 